\title{
Silicene is a phantom material
}

\author{
E. F. Sheka \\ Department of Theoretical Physics and Mechanics \\ Peoples' Friendship University of Russia, \\ Str. Miklukho-Maklay, 6, Moscow 117198, Russia \\ sheka@icp.ac.ru
}

DOI 10.17586/2220-8054-2016-7-6-983-1001

\begin{abstract}
The paper presents a comparative consideration of $s p^{2}$ nanocarbons and their silicon and higher tetrels analogues from the viewpoint of the spin molecular theory taking into account the electron correlation in open-shell molecules. High radicalization of silicene and quantum instability of flat honeycomb 2D structures of germanene and stanene make all the species phantom materials leaving graphene the only one-atom thick 2D solid free of the crucial restrictions.
\end{abstract}

Keywords: silicene, siligraphene, boronitrosilicene, spin molecular theory, unrestricted Hartree-Fock formalism, open-shell molecules.

Received: 30 May 2016

\section{Introduction}

Silicene holds a special place in graphene science. This phantom material was called into being by an unprecedented activity of the graphene science development, while not of the science itself, but of its theoreticalcomputational part predominantly. Actually, the first appeal to silicene took place even before the 'graphene era' in 1994 [1] when looking for Si and Ge analogues of graphite. Since the sought-for materials did not exist (as well as they have not existed until now) the study was restricted to the theoretical consideration. Evidently, one-atom thick monolayer of the Si- (and Ge-) graphite of presumably $s p^{2}$ electron configuration was the mainly studied model. Because of hexagonal honeycomb structure of the layers, the prediction of Dirac cones attributed to $\pi$ bands at $\mathrm{K}$ points of the Brillouin zone was quite expected. This was the point from which a close similarity between graphene and its virtual heavier-tetrel analogues started. The repeated reference to the subject took place 13 years later [2] when the term silicene was introduced to describe virtual free standing one-atom thick honeycomb monolayer consisting of silicon atoms. In the frame of tight-binding approximation, a description of silicene was suggested in terms of Dirac fermions similarly to the one described for graphene. The study was the beginning of a large stream of theoretical and computational studies, implementing and securing representation in the scientific community about a new highly promising material (see reviews [3-8] and references therein).

The era of real silicene started in 2008 when a possibility to obtain strips of adsorbed silicon atoms on $\operatorname{Ag}(110)$ surface was firstly announced [9]. A new and attractive word silicene was largely used across the publication, though no one-atom honeycomb free-standing structure was fixed. Just as a response to the publication, a quantumchemically grounded skeptical view on silicene's existence was suggested [10]. As if supporting this conclusion and making sure that the free-standing silicene cannot be obtained, experimenters have focused their efforts on obtaining hexagon-packed structure of silicon atoms monolayers on different surfaces. By 2012 a few groups had succeeded in experimental observations of the sought silicon monolayers obtained in due course of epitaxial growth in ultra high vacuum on different surfaces of crystalline silver [11-14] and diboride [15]. Successful experiments on Ir [16] and other substrates (see a comprehensive review [17]) then followed. The produced monolayers are tightly connected with substrates and are rightly referred to as epitaxial silicene [1]. The monolayers behave as typical objects of surface science, subordinating to crystallography of substrates in the best way and undergoing phase transitions when the substrate crystallography favors them.

Epitaxial silicene demonstrates some properties, such as the availability of Dirac cones in the electronic bands at Fermi level $[11,14]$ and lays the foundation of the report on the silicene field-effect transistor due to growth-transfer-fabrication process that was devised via epitaxial silicene encapsulated delamination with native electrodes [18]. However, these features are mostly related to the physics of adlayers on different substrates, which is rich and highly variable but has no connections with that demonstrated by real free standing graphene sheets. Therefore, remaining in the field of graphene science, one should join for a common discussion theoretical consideration of graphene and virtual silicene only. And the first question which must be answered is: Why free standing silicene cannot exist? The current paper is aimed at answering the question on the basis of clarifying 
what we know about spin effects in $s p^{2}$ nanosilicons and relativistic electrons of silicene and what the difference is between the latter ones and those of graphene.

\section{Dirac Fermions in 2D Silicene Crystal}

Theoretical investigations of silicene have been presented in hundreds of publications. However, paradoxically they cover a rather narrow range of topics. The first thing to note concerns a complete ignoring (except two papers $[10,19])$ crystalline-molecular dualism that is so picturesque in graphene and forms two streams in the graphene theoretical investigations, dividing them into solid state and molecular ones. In the case of silicene, there is solely the first one, moreover, with a substantial predominance of Dirac's fermions concept. Evidently, it is due to a desire to see properties in silicene that may be useful when applied in electronics and spintronics. Addressing quantum chemical calculations, it is the reason for a particular preference of periodical boundary conditions (PBC) in constructing models for further computational studying within the DFT approach mainly, involving its tight-binding version.

Because the Si-Si interatomic distance is larger than that of C-C, $\pi-\pi$ (better to say $p_{z}-p_{z}$ ) overlap weakens due to which two DFT PBC honeycomb configurations are usually considered, namely, planar and buckled shown in the top of Fig. 1. $\pi$ Bands structures in the bottom of the figure are obtained by using ab initio calculations [21]. As in the case of graphene, $\pi$ bands of silicene involve cones in the vicinity of the Fermi level at energy $E_{f}$ which is a consequence of hexagon symmetry of the considered honeycomb structures. In the absence of spin-orbit coupling (SOC), the bands in this region are described by the Dirac-like Hamiltonian $\hbar v_{F} \boldsymbol{\sigma} \cdot \boldsymbol{\kappa}$. However, the SOC in silicene is quite considerable due to significant intrinsic SOC in silicon atoms. Accordingly, the quasirelativistic Hamiltonian is modified by including SOC and takes the form [20]:

$$
\hat{H} \approx\left(\begin{array}{cc}
-\xi \sigma_{z} & v_{F}\left(k_{x}+i k_{y}\right) \\
v_{F}\left(k_{x}+i k_{y}\right) & \xi \sigma_{z}
\end{array}\right),
$$

where $v_{F}$ is the Fermi velocity of $\pi$ electrons near the Dirac points with nearly linear energy dispersion, $\sigma_{z}$ is Pauli matrix and $\xi$ presents the effective SOC. The above equation results in the spectrum:

$$
\left.E(\boldsymbol{\kappa})= \pm \sqrt{\left(v_{F} k\right)^{2}+\xi^{2}}\right)
$$

Therefore, one can estimate the energy gap, which is $2 \xi$ at the Dirac points and is of $0.07 \mathrm{meV}$ and 1.55 meV for planar and buckled silicene, respectively. Such presentation of the low-energy band structure has been widely accepted in general, although repeated calculations disclose some quantitative variations.

Much effort has been invested to experimentally observe Dirac fermions (see rev. [7] and references therein). At the same time it was not about a confirmation of theoretical consideration, since it does not concern the difference between virtual and epitaxial silicene that was obvious. The insisting attempts were challenged by the fact that the Dirac cones are a consequence of hexagonal symmetry of one-atom thick layers which could be expected in the case of epitaxial silicene as well. Actually, a similar packing in monolayers of adsorbed atoms were not so rare in surface science when a proper substrate was selected. The expectations came true and the Dirac cones were observed for monolayers of silicon atoms at Ag (111)-(1x1) surface by angle-resolved photo-electron spectroscopy (ARPES) [11] and quasiparticle interference (QPI) [14]. The Ag(111) surface happens to be the most suitable for hosting regular structures of atomic silicon adsorbate. Apparently it is coherent with the regular hexagon-packed adlayers of silver on $\mathrm{Si}(111)-(1 \mathrm{x} 1)$ surface that were the beloved objects of the surface science.

Fig. 2 exhibits the Dirac cone obtained by ARPES [11]. A clearly seen energy gap of $0.3 \mathrm{eV}$ was observed. The value greatly exceeds that caused by the SOC and points to a strong substrate influence. The problem of the substrate still remains a contested point hindering the justification for applying the quasirelativistic Dirac fermion concept to the description of epitaxial silicene [17]. This is particularly the case due to the still unmet expectations of the spin Hall Effect the observation of which at suitable temperatures is favored by significant SOC.

\section{Spin Effects of Silicon-Based Open-Shell Molecules}

\subsection{Peculiarities of the $N_{D}(R)$ Graphs of $\mathrm{C}=\mathrm{C}, \mathrm{Si}=\mathrm{C}$, and $\mathrm{Si}=\mathrm{Si}$ Bonds}

In order to better understand the spin effects of silicene molecules, we will address the cluster (molecular) approach to the silicene model structure instead of PCB duplication of primitive unit cells so that possible quantum instability of low-dimensional systems can be avoided [22]. Next, the standard closed-shell DFT formalism, which is mainly used and which is practically unable to disclose spin effects, will be substituted by the unrestricted Hartree-Fock (UHF), which has established itself as a very effective means of detecting delicate spin features of molecules $[23,24]$. The effects determine the characteristics for the open-shell character of the latter and 

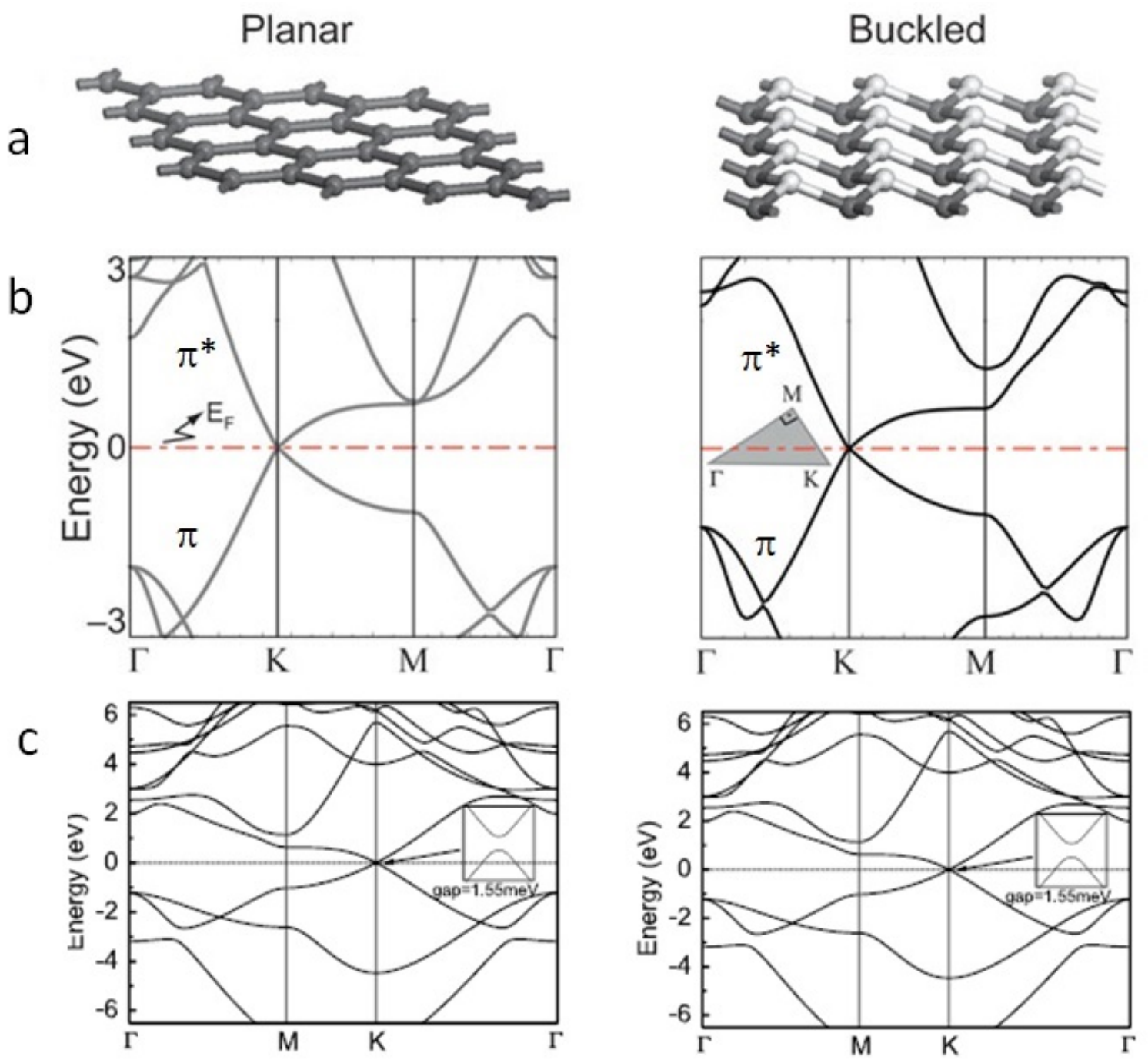

FIG. 1. (a),(b). Geometry and band structure of flat and buckled DFT PBC silicene (adapted from [21]). (c) Band structure involving SOC (adapted from [20])

are described by spin contamination of their electronic states that causes the availability of effectively unpaired electrons of $N_{D}$ total number. Concerning $s p^{2}$ siliceous molecules, we meet the spin effects first when looking for disilene molecules. Based on the data of a scrupulous study of the issue [25], Fig. 3 presents $N_{D}(R)$ graphs related to the dissociation of ethylene, silaethylene (or silaethene), disilene and disilane molecules. Comparing the graphs for $\mathrm{C}=\mathrm{C}$ and $\mathrm{Si}=\mathrm{Si}$ bonds, a drastic difference becomes evident. In the case of ethylene, one can distinctly see that $\pi$ electrons govern the molecule's continuous dissociation when the interatomic distance changes from $1.4 \AA$ to $1.9 \AA$ and then comes the turn of $\sigma$ electrons until the dissociation is completed at $2.8 \AA$. At equilibrium the molecule is a closed-shell one with the $\mathrm{C}=\mathrm{C}$ bond of $1.326 \AA$ in length. Oppositely, $\pi$ electrons are practically unobservable under disilene dissociation since already in equilibrium they are almost fully transformed into a pair of effectively unpaired electrons $\left(N_{D}=1.78 \mathrm{e}\right)$. Therefore, disilene has no closed-shell phase at all and in the equilibrium state is open-shell one. Essentially similar but quantitatively different is the situation for the $\mathrm{Si}=\mathrm{C}$ bond of silaethylene. As seen in the figure, at equilibrium, the molecule is open-shell one, the radicalization of which requires $0.153 e$.It is well documented that both silaethylene and disilene molecules are highly reactive and their stabilization usually occurs at low temperatures in rare gas matrices [26].

Since the $\pi$ electrons of silaethylene and disilene are highly dissociated, the valence electron hybridization is evidently not $s p^{2}$ one. This is consistent with the previouh conclusion that because of the large size and the inner-shell electron's shielding in silicon atoms, the overlap between $p_{z}$-orbitals in the relevant molecules is negligibly small, and thus tends to not form $\pi$-bonds preferentially [27]. This feature greatly influences the valence 

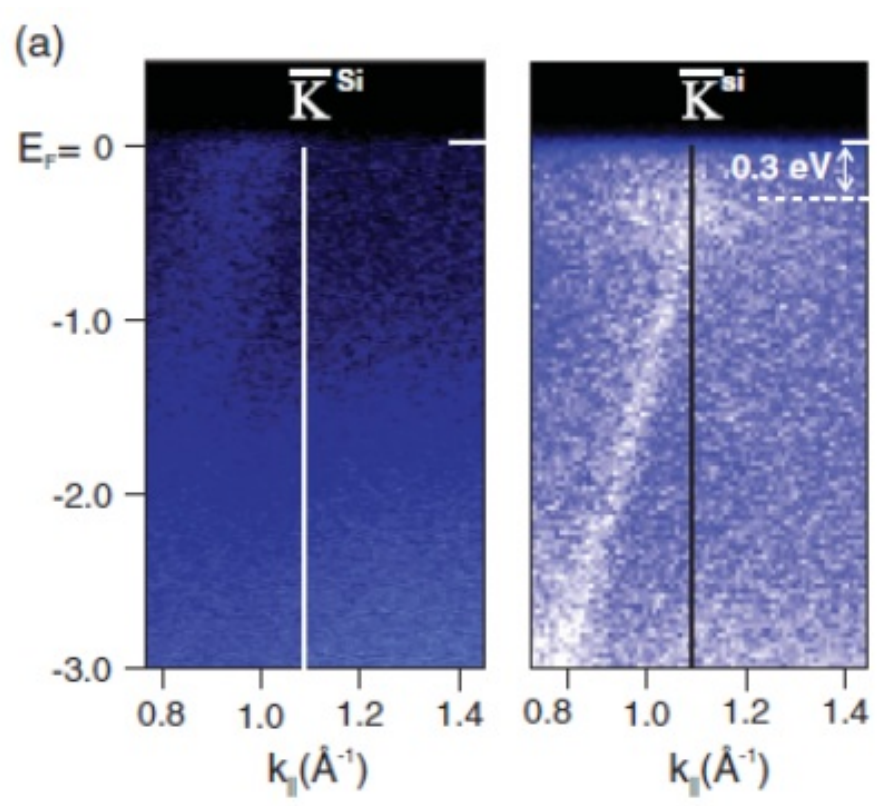

(b)

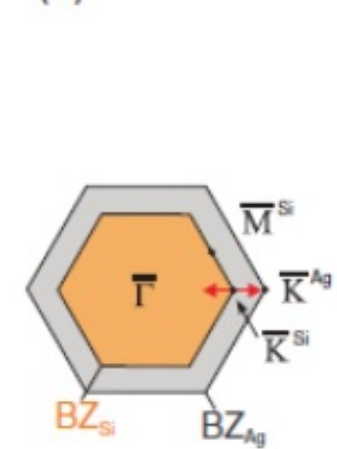

FIG. 2. (a) Angle-resolved photo-electron spectroscopy intensity map for the clean Ag surface (left) and after formation of the 2D Si adlayer (right), taken along the $\vec{\Gamma} \cdot \vec{K}^{A g}$ direction through the silicene $\vec{K}^{S i}$ point $(h \nu=126 \mathrm{eV})$. (b) Brillouin-zone scheme of the 2D Si layer with respect to the $\operatorname{Ag}(111)-(1 \times 1)$ surface. The red arrow indicates the ARPES measurement direction (adapted from [11])

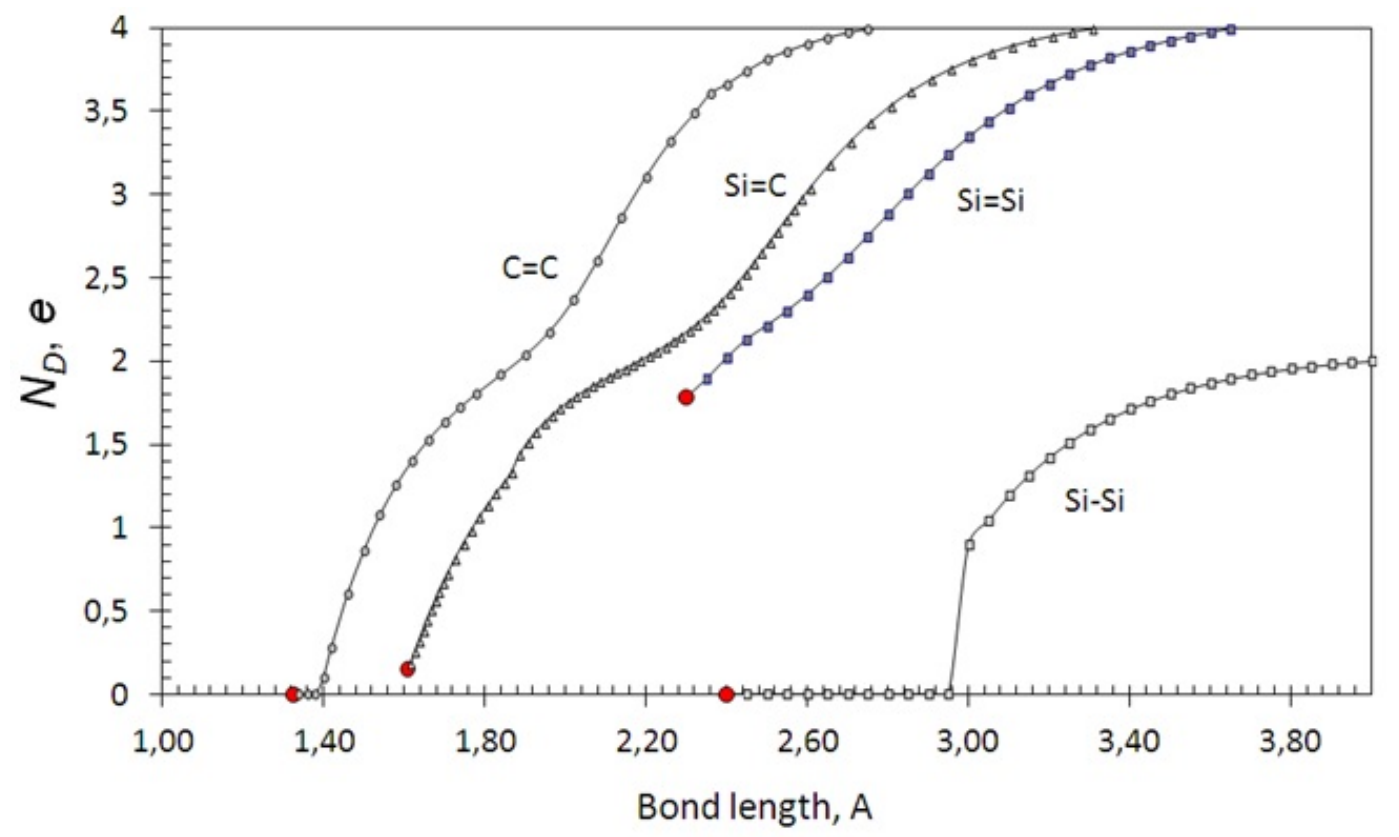

FIG. 3. $N_{D}(R)$ graphs related to the dissociation of double $\mathrm{C}=\mathrm{C}, \mathrm{Si}=\mathrm{C}$ and $\mathrm{Si}=\mathrm{Si}$ as well as single Si-Si bonds. Big balls mark the bonds in the equilibrium state of ethylene, silaethylene, disilene and disilane molecules, respectively. UHF AM1 calculations 
electrons' hybridization, changing it from $s p^{2}$ for carbon atoms to $s p^{3}$-like for silicon [28] when $p_{z}$ electrons become effectively unpaired. Consequently, the number of effectively unpaired electrons presents a measure of the transition between the two hybridization modes. When the value per atom, namely, $N_{D A}$, is zero, the relevant atoms retain their $s p^{2}$ mode. When $N_{D A}$ is unity one should speak about a complete unpairing of $p_{z}$ electrons and of $s p^{3}$ hybridization with the only difference that the feature concerns not valence-terminated atoms (as in the case of disilane) but radicals. Within this range one can speak of $s p^{3}$-like hybridization that gradually strengthens while $N_{D A}$ increases from zero to unity. In the case of ethylene the equilibrium-state $N_{D A}$ is zero, which provides a clear vision of the $s p^{2}$ hybridization of the molecule atoms. In disilene, $N_{D A}=0.89 e$, which is quite close to unity, which amply explains why the equilibrium $\mathrm{Si}-\mathrm{Si}$ distance in this case $(2.30 \AA)$ is close to that of disilane $(2.40 \AA)$.

\subsection{Silicon Analogues of $\mathbf{s p}^{2}$ Carbonaceous Molecules}

To make the picture more complete, the results obtained for siliceous molecules will be discussed alongside with those related to the core-equivalent carbon analogs. The data of a complete family of such-selected Xcompounds $(\mathrm{X}=\mathrm{C}, \mathrm{Si})$ is presented in Table 1 . The number of odd electrons $N$ counts all valence electrons that do not participate in the formation of $\sigma$ bonds; $\Delta E^{R U}=E^{R H F}-E^{U H F}$ presents the difference of energies related to closed-shell (restricted) and open-shell (unrestricted) odd electron configurations. Eight different molecules were compared, among which there are $\mathrm{X}$-benzene, fullerene $\mathrm{X}_{60}$, fragments of $(6,6)$ and $(10,0)$ single-walled $\mathrm{X}$-nanotubes, with both empty and hydrogen-terminated end atoms, and $(3,7) \mathrm{X}$-hexagon flat fragments with two types of the edgeatom termination. The computations have revealed a high similarity in the shape of equilibrium structures of the molecules with the only difference concerning the length of the relevant $\mathrm{C}=\mathrm{C}$ and $\mathrm{Si}=\mathrm{Si}$ bonds. As for $\Delta E^{R U}$ and $N_{D}$ parameters, the first one is a consequence of the spin-dependent splitting of the degenerate restricted Hartree-Fock (RHF) states and is given in the table in absolute and percentage values, the latter given in parentheses with respect to the $E^{R}$ energy. The second parameters present the total number of effectively unpaired electrons $N_{D}$ and the doubled spin contamination since due to the numbers of spins $\alpha$ and $\beta$ and are equal, $N_{D}=2 \Delta \hat{S}^{2}$. In parentheses is given roughly averaged value, $\alpha$ obtained by just dividing $N_{D}$ to the number of $\mathrm{X}$-type atoms involved. The actual distribution of the value over the molecule's structure is quite irregular (see numerous examples in [23]) and should be calculated for each molecule individually.

The data listed in Table 1 exhibit a tremendous difference in the data for carbon and silicon molecules caused by the difference in the correlation of their $p_{z}$ electrons. Thus, in the carbon family the electron correlations and transformations from closed-shell to open-shell molecules have exhibited themselves starting from fullerene $\mathrm{C}_{60}$, while benzene molecule is well defined closed-shell species. In the series of condensed aromatics, the electron correlation becomes visible in naphthalene and strengthens when the molecule size increases [29]. Thus nature seems to let benzene play a particular role for establishing and proving the aromaticity concept as well as for introducing $\pi$ electrons in organic chemistry. In contrast to benzene, fullerene $\mathrm{C}_{60}$, as well as the studied fragments of carbon nanotubes and graphene belongs to the open-shell class of molecules and exhibit quite strong electron correlation. This very effect explains unique peculiarities of the species concerning their chemistry, magnetism, and biomedical behavior that have been discussed in detail previously [23]. At the same time, the $p_{z} N_{D}$ values fill the interval $(0.17 e-0.39 e)$ due to which the species are not completely radicalized and the atom hybridization, though $s p^{3}$-like one, is close to $s p^{2}$. Both these features account for the production and existence of the carbon species under ambient conditions.

In contrast to the above case, all the quantities related to the silicon counterparts clearly highlight a very strong electron correlation and the atom hybridization close to the radical $s p^{3}$ one in all the cases This results in practically complete radicalization of the species. Actually, as seen in the table, the $N_{D}$ values are equal to or even slightly exceed the number of odd electrons $N$ in all the cases making molecules $N$-fold radicals. A complete radicalization of the species evidently prevents from their production and existence at ambient conditions. A schematic view of the difference between carbon and silicon valence deficient compounds, presented in Fig. 4 on the background of the graph of ethylene, clearly shows the difference between the two families of species.

Therefore, the silicon-based open-shell molecules exhibit tremendous spin effects that are consequence of a strong correlation of their $p_{z}$ electrons, on the one hand, and cause high radicalization of the molecules as whole, on the other [29]. The last feature is the main reason that prevents one from obtaining these species experimentally. As for computational description, the open-shell character of the molecules mandatorily requires their consideration in the framework of the CI theory. While the UHF formalism covers CI effects virtually [30] the DFT (including UDFT) is quite insensitive to the latter. At the same time, most published computational results have been obtained when using one of the available DFT closed-shell versions thus making the performed considerations unrealistic. 
TABLE 1. Equilibrium structures of silicon-based molecules and $\Delta E^{R U}$ and $N_{D}$ parameters of the equi-X-core $(\mathrm{X}=\mathrm{C}, \mathrm{Si})$ molecules; number of odd electrons $N$ and effectively unpaired $N_{D}$ in $e$; energies in $\mathrm{kcal} / \mathrm{mol}$

Molecules

(UHF calculations)
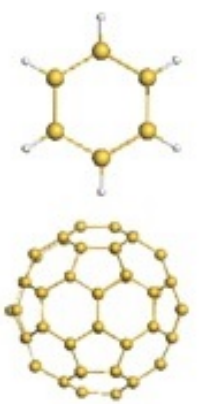

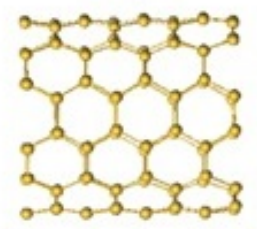

0

a a a a

$\int_{0-0}^{\infty} a_{0}^{2} a_{0}^{2}$

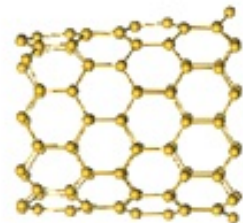

की

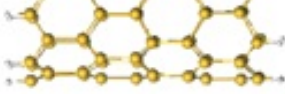

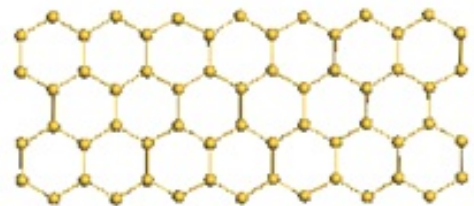

ida d i a d bo

a ca a o a a a

ato a a a a da

de a a a a a o

\begin{tabular}{|c|c|c|c|c|c|}
\hline \multirow{2}{*}{$\begin{array}{l}\text { Chemical } \\
\text { formula }\end{array}$} & \multirow{2}{*}{$N_{\text {odd }}$} & \multicolumn{2}{|c|}{ Carbon } & \multicolumn{2}{|c|}{ Silicon } \\
\hline & & $\begin{array}{c}\Delta E^{R U} \\
(\%)\end{array}$ & $\begin{array}{c}N_{D} \\
\left(N_{D A}^{a v}\right)\end{array}$ & $\begin{array}{c}\Delta E^{R U} \\
(\%)\end{array}$ & $\begin{array}{c}N_{D} \\
\left(N_{D A}^{a v}\right)\end{array}$ \\
\hline $\mathrm{X}_{6} \mathrm{H}_{6}$ & 6 & $\begin{array}{c}0 \\
(0)\end{array}$ & $\begin{array}{l}0 \\
(0)\end{array}$ & $\begin{array}{l}23.26 \\
(16.1)\end{array}$ & $\begin{array}{c}2.7 \\
(0.45)\end{array}$ \\
\hline$X_{60}$ & 60 & $\begin{array}{l}17.26 \\
(1.8)\end{array}$ & $\begin{array}{c}10 \\
(0.17)\end{array}$ & $\begin{array}{l}282.69 \\
(21.8)\end{array}$ & $\begin{array}{c}62 \\
(1.03)\end{array}$ \\
\hline \multirow{2}{*}{$\begin{array}{c}(6,6) \\
\text { armchair } \\
X- \\
\text { nanotube }\end{array}$} & 120 & $\begin{array}{c}133.85 \\
(6.5)\end{array}$ & $\begin{array}{c}43 \\
(0.36)\end{array}$ & $\begin{array}{l}759.28 \\
(30.0)\end{array}$ & $\begin{array}{c}128 \\
(1.07)\end{array}$ \\
\hline & 96 & $\begin{array}{l}103.0 \\
(15.9)\end{array}$ & $\begin{array}{c}25 \\
(0.21)\end{array}$ & $\begin{array}{r}415.37 \\
(21.4)\end{array}$ & $\begin{array}{c}96 \\
(0.80)\end{array}$ \\
\hline \multirow{2}{*}{$\begin{array}{c}(10,0) \\
\text { zigzag } \\
X- \\
\text { nanotube }\end{array}$} & 120 & $\begin{array}{l}499.58 \\
(21.2)\end{array}$ & $\begin{array}{c}44 \\
(0.37)\end{array}$ & $\begin{array}{l}854.06 \\
(30.2)\end{array}$ & $\begin{array}{c}115 \\
(0.96)\end{array}$ \\
\hline & 100 & $\begin{array}{c}274.14 \\
(30.6)\end{array}$ & $\begin{array}{c}30 \\
(0.25)\end{array}$ & $\begin{array}{l}538.83 \\
(25.4)\end{array}$ & $\begin{array}{c}100 \\
(0.83)\end{array}$ \\
\hline \multirow{2}{*}{$\begin{array}{c}(7,3) \\
X- \\
\text { nanosheet }\end{array}$} & 82 & $\begin{array}{c}386.62 \\
(20.5)\end{array}$ & $\begin{array}{c}32 \\
(0.39)\end{array}$ & $\begin{array}{l}590.76 \\
(30.3)\end{array}$ & $\begin{array}{c}76 \\
(0.93)\end{array}$ \\
\hline & 60 & $\begin{array}{l}113.38 \\
(34.6)\end{array}$ & $\begin{array}{c}15 \\
(0.18)\end{array}$ & $\begin{array}{c}252.12 \\
(20.1)\end{array}$ & $\begin{array}{c}56 \\
(0.68)\end{array}$ \\
\hline
\end{tabular}




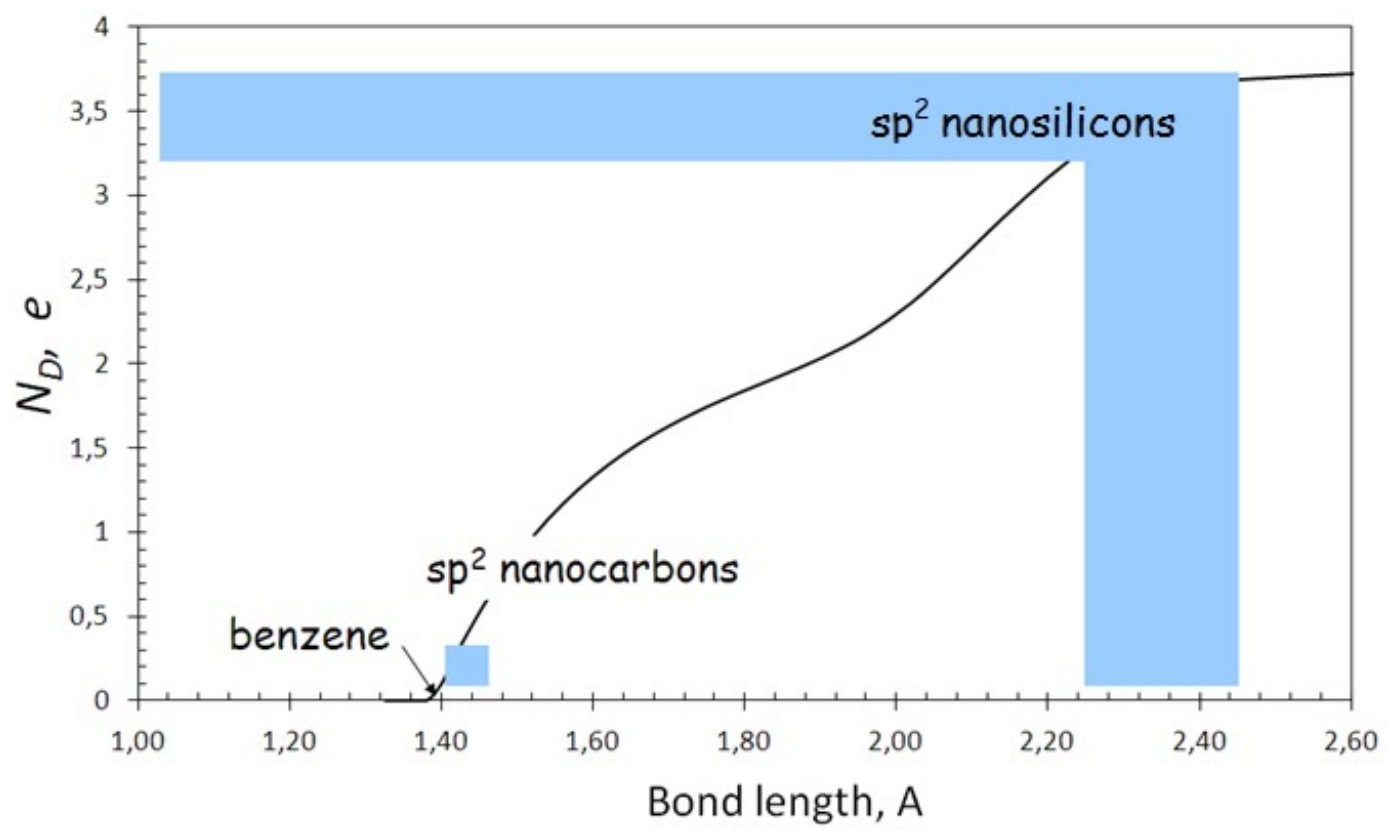

FIG. 4. A schematic presentation of the difference in the $N_{D}(R)$ graphs of $s p^{2}$ nanocarbons and nanosilicons

\section{Last News on New One-Atom Thick 2D Silicene-Based Material $\mathrm{Si}_{2} \mathrm{BN}$}

The use of widely-accessible DFT PBC techniques has led to a virtually endless stream of publications claiming prediction of more and more 'new 2D materials' with a number of attractive properties (see [22] and references therewith, but a few) The latest, but certainly not to be the last one was discussed on Ray Kurzweil's Accelerating Intelligence website on March 25, 2016. It concerned the prediction of a new one-atom thick $2 \mathrm{D}$ material $\mathrm{Si}_{2} \mathrm{BN}$ made of light inexpensive earth-abundant elements boron, nitrogen, and silicon "that might push graphene into the background". The news was accompanied with a PR movie that is still rare in practice, but certainly points to the emergence of a new trend in the presentation of scientific results directly from the authors. The material in question is based on silicon and exactly speaking presents a boronitrosilicene As in the majority of similar cases, it is predicted computationally and the authors appeal to a conventional, gentlemen's set, including flat structure, inherent metallicity, easy flexibility high strength and presumably conductivity for heralding their discovery of "a new class of single-atom-thick graphenelike material formed from $\mathrm{Si}_{2} \mathrm{BN}$ with unusual characteristics using $a b$ initio simulations" [31]. The authors are absolutely convinced in their discovery and are not at all embarrassed by the fact that their pristine silicene is a virtual material. Apparently it should be assumed that in the framework of theoretical concepts shared by them that silicene is as real as graphene.

One could only rejoice in this power of faith in the omnipotence of quantum-chemical calculations, if only errors were not made by the authors in their calculations. Since similar errors occur frequently and tend to build on one another, it is worthwhile to consider them in detail, and in this case, $\mathrm{Si}_{2} \mathrm{BN}$ is a good example to clearly illustrate this point. As mentioned earlier, the first trouble awaits computational experts when constructing the model structure of the species in question. Following the work of others, the authors [30] used PBC to duplicate a selected unit cell for getting an extended regular structure fully ignoring possible quantum instability of lowdimensional structures [21], which impugn the PBC application. In the case of covalent structures, involving different covalent bonds, in particular, one should be particularly careful. Thus, in the case of $\mathrm{Si}_{2} \mathrm{BN}$ one deals with four types of covalent bonds, namely: $\mathrm{Si}=\mathrm{Si}, \mathrm{Si}=\mathrm{B}, \mathrm{Si}=\mathrm{N}$, and $\mathrm{B}-\mathrm{N}$. Not all the bonds are quite stable. Unfortunately, not much is known about the three double bonds. However, judging by the relevant single bonds, one might expect considerable instability for the $\mathrm{Si}=\mathrm{B}$ bonds only. Actually, highly stable structures are characteristic for crystalline silicon and silicon nitrides while silicon boride's crystal is metastable, primarily due to the instability of Si-B bonds [31]. The authors of the discussed paper [30] met this trouble when composing the unit cell for $\mathrm{Si}_{2} \mathrm{BN}$ : only one of three compositions retained planar structure in the course of the further optimization. This fact should have warned the authors of the fullest confidence in the stability of the PBC-replicated structure. As will be shown below, this unease was confirmed. 
The second issue concerns the calculation technique used, which as usual was one of standard versions of closed-shell DFT theory. To be confident in the correctness of the techniques application it is necessary to verify that the studied system is a closed-shell one. Graphene and silicene are open-shell systems, which is caused by length of the relevant $\mathrm{C}=\mathrm{C}$ and $\mathrm{Si}=\mathrm{Si}$ bonds [25]. Since the latter constitute the majority of the $\mathrm{Si}_{2} \mathrm{BN}$ species another situation should be hardly expected in this case. The correlated open-shell systems require UHF or other CI technique for their consideration.

Following the computational study of silicene, which was discussed in detail in Section 3, let us consider $\mathrm{Si}_{2} \mathrm{BN}$ from the viewpoint of the UHF calculations. Outlining that $\mathrm{Si}_{2} \mathrm{BN}$ is based on the silicene honeycomb structure it would be useful for the future to mark it as $s-\mathrm{BNSi}_{2}$ (as used for graphene-like materials, such as siligraphenes see the next Section). Presented in Fig. 5a displays the $(5,5) s-\mathrm{BNSi}_{2}$ regular structure (the molecule presents a right-angled sheet with five benzenoid-like units along armchair and zigzag edges, respectively) that is coherent with the working model considered in [31]. The molecule is flat and the atom distribution is governed by chemical bonds $\mathrm{Si}=\mathrm{Si}$ of $2.29 \AA ; \mathrm{Si}=\mathrm{B}$ of $1.62 \AA ; \mathrm{Si}=\mathrm{N}$ of $1.54 \AA$; and $\mathrm{B}-\mathrm{N}$ of $1.44 \AA$. The first three bonds correspond to the UHF-equilibrated molecules $\mathrm{H}_{2} \mathrm{Si}=\mathrm{SiH}_{2} ; \mathrm{H}_{2} \mathrm{Si}=\mathrm{BH}$; and $\mathrm{H}_{2} \mathrm{Si}=\mathrm{NH}$, respectively. The edge silicon atoms are terminated by hydrogens. As seen in Fig. 5b, subjected to further optimization, the structure retains its flat geometry but losses honeycomb packing and becomes irregular thus becoming a perfect exhibition of quantum instability for the the PBC structure considered in [31]. The same relates to fragments of the pristine $(5,5) s-\mathrm{BNSi}_{2}$ involving quasi-benzene, quasi-naphthalene and quasi-pentacene. None of these fragments preserves the hexagonal quasibenzenoid structure involving simultaneously $\mathrm{Si}=\mathrm{Si}, \mathrm{Si}=\mathrm{N}$, and $\mathrm{Si}=\mathrm{B}$ bonds.

To ascertain if this instability is caused by either the three-set bond group or silicon atoms entering each of the bonds, the analogous graphene $(5,5) \mathrm{g}-\mathrm{BNC}_{2}$ molecule, composed in the same manner as $s$-BNSi $\mathrm{B}_{2}$, was analyzed. Fig. $5 \mathrm{c}$ presents the equilibrium structure of the molecule while Fig. $5 \mathrm{~d}$ exhibits the state of its radicalization in view of the $N_{D A}$ map that exhibits the distribution of $N_{D}$ effectively unpaired electrons over the molecule's atoms. The main pattern of the map is provided by carbon atoms excluding two top and two bottom nitrogen atoms presented by their dangling bonds. The map gives evidence for both the availability of local spins at the carbon atoms and their differing values at different atom locations which reveals a peculiar distribution of $\mathrm{C}=\mathrm{C}$ bond lengths over the molecule.

In view of the difference between equilibrium structures of the $(5,5) s$ - $\mathrm{BNSi}_{2}$ and $(5,5) g$ - $\mathrm{BNC}_{2}$ molecules it is possible to conclude that the breakdown of the former is caused by the chemical bonds silicon atoms being too long to be accommodated by shorter $\mathrm{Si}=\mathrm{B}, \mathrm{Si}=\mathrm{N}$, and $\mathrm{B}-\mathrm{N}$ bonds to provide a regular honeycomb structure. Not considering these chemical features leads to erroneous heralding of 'new materials' that cannot exist even virtually. Unfortunately, such errant statements on the DFT-based PBC predictions of new materials in the current graphenics have become more commonplace In view of this it is worthwhile to remember bitter words of Roald Hoffmann in his notes on "Small but Strong Lessons of Chemistry in Nanoscience" [33]: "It is clear that quantum-mechanical modeling of molecules and extended structures has become easy to do. Much too easy, I would say... What worries me more (and just what you would expect from me ...) is that the calculations are done in a way blindly, with no calibration on related chemistry, no controlling checks with chemical intuition".

\section{Silagraphenes}

Another phantom material related to $s p^{2}$ hybridized silicon atoms concerns the recently heralded 'new materials' called siligraphenes [34-36]. Adjusting the new nomenclature with that previously used in organosilicon chemistry, it would be better to call these compounds siligraphenes similar to silaethylene, silaalkane, and so forth. As in the case of boronitrosilicene, silagraphenes were created computationally by using a DFT PBC approach. The main idea was to place the silicon and carbon atoms constituting the cubic spatial structure of silicon carbide in one plane. Thus, by modulating $\mathrm{Si}=\mathrm{C}$ bond lengths flat configurations of silagraphenes $g$-Si $\mathrm{C}_{x} \mathrm{C}_{1-x}$ with $x$ as the governing parameter [35], $g$ - $\mathrm{SiC}_{1}$ and $g-\mathrm{SiC}_{3}$ [34] and $g-\mathrm{SiC}_{7}$ [36] were constructed. And again, the indispensable and failsafe DFT used in its standard versions related to closed-shell approximation supplemented by PBC readily issued a positive conclusion concerning the models: silagraphenes are stable graphene-like flat $2 \mathrm{D}$ materials of honeycomb-like structure, the latter being slightly irregular due to the difference between the $\mathrm{C}=\mathrm{C}$ and $\mathrm{Si}=\mathrm{C}$ bond lengths. Thus, the obtained new materials are characterized by typical spectra of the Dirac fermions. Meeting the next temptation of a possible tuning of the conical energy spectrum, the Si:C content ratio was suggested as a desired modulator. The full readiness of silagraphenes applicability to advanced electronics and spintronics was heralded.

The example discussed in the previous section compels us to treat these optimistic statements with caution. Fig. 6 presents a comparative view on isostructural silagraphenes and pristine graphene on the basis of $(5,5)$ nanographene (NGr) in the UHF formalism framework. The panels on the right of the figure show the equilibrium 
$(5,5) s-\mathrm{BNSi}_{2}$ start

(a)

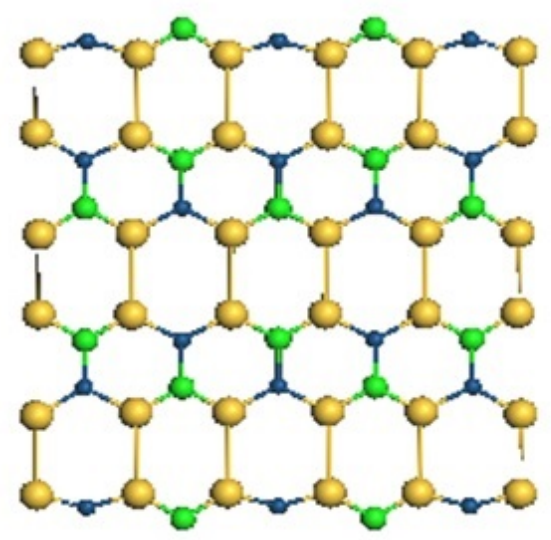

(c) $(5,5) g-\mathrm{BNC}_{2}$ opt

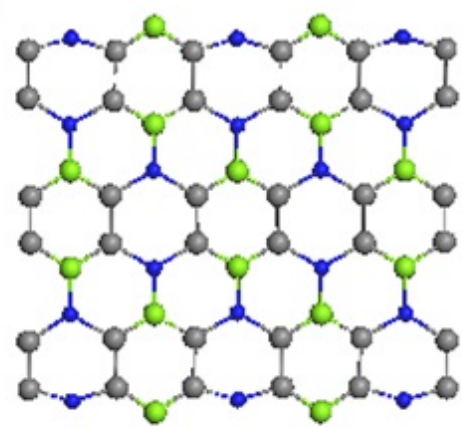

$\mathrm{N}_{\mathrm{D}}=14.088 e$
$(5,5) s-\mathrm{BN} \mathrm{Si}_{2}$ opt

(b)
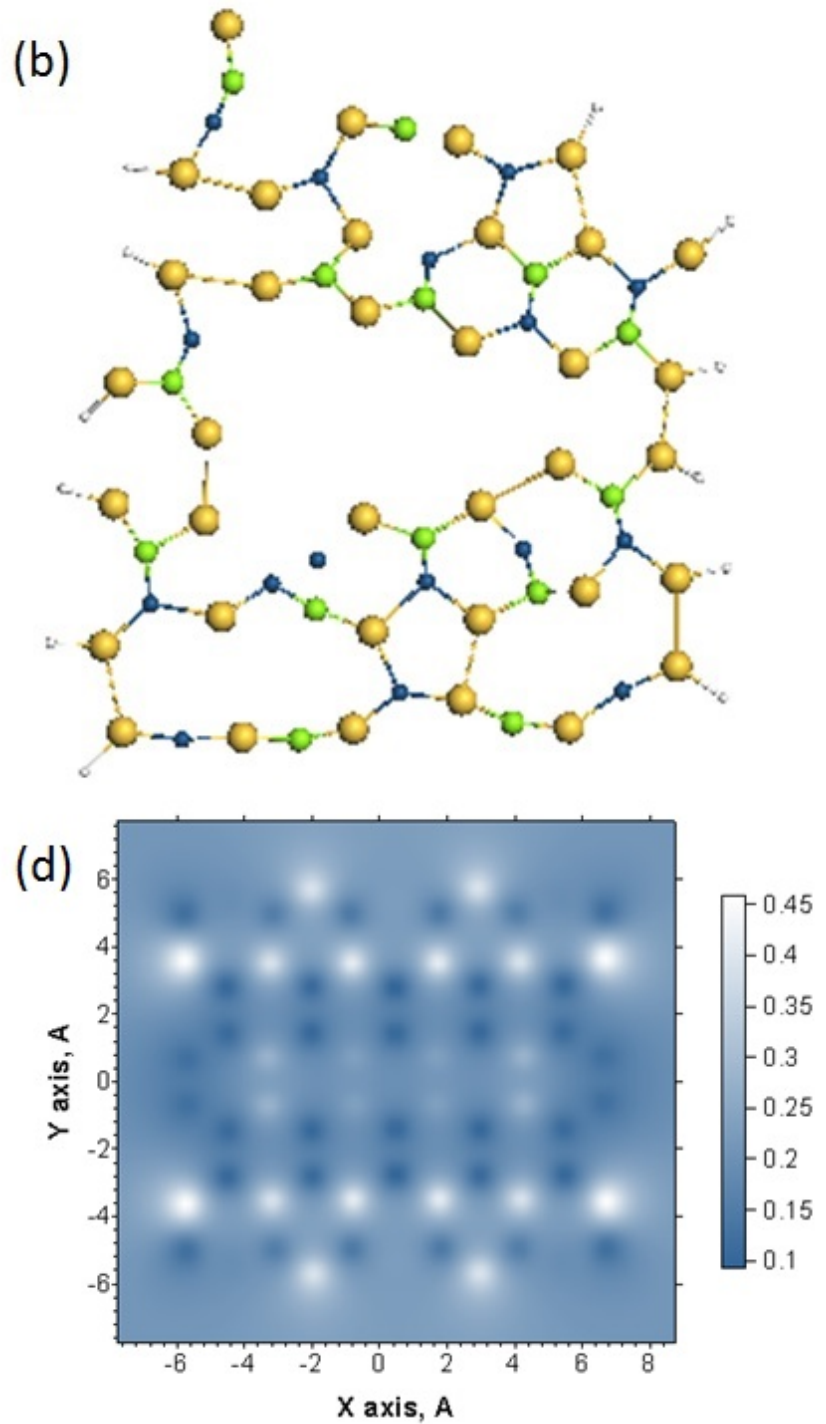

FIG. 5. Start (a) and optimized (b) structures of the $s$-BNSi ${ }_{2}$ molecule; (c) optimized structure and (d) $N_{D A}$ map of the effectively unpaired electrons (local spins) distribution of $g$-BNC 2 molecule. Hydrogen atoms terminating edge silicon (a) and carbon (c) atoms are not shown. AM1-UHF calculations. Big and small light-grey, dark gray and small dark balls mark silicon, nitrogen, carbon and boron atoms, respectively

structures for three molecules, namely: $(5,5) \mathrm{NGr},(5,5) g-\mathrm{SiC}_{3}$ and $(5,5) g-\mathrm{SiC}_{7} . \mathrm{Si}=\mathrm{C}$ and $\mathrm{C}=\mathrm{C}$ bond lengths lay in the intervals of $\{1.76-1.66\} \AA$ and $\{1.44-1.23\} \AA$ in $g-\mathrm{SiC}_{3}$ while of $\{1.73-1.66\} \AA$ and $\{1.48-1-27\} \AA$ in $g-\mathrm{SiC}_{7}$. All $\mathrm{Si}=\mathrm{C}$ bonds are longer than the equilibrium length in the silaethylene molecule which constitutes 1.60 $\AA$ and provides a considerable radicalization of the molecule as seen in Fig. 3. Therefore, the silicon components of both silagraphenes are obviously radicalized pointing to the open-shell character of the molecules electronic states. The carbon component retains its open-shell character as well since there are several $\mathrm{C}=\mathrm{C}$ bonds longer than $1.395 \AA$. At the same time, some of the $\mathrm{C}=\mathrm{C}$ bonds of the pristine graphene remarkably shorten, which is caused by the accommodation of the neighboring $\mathrm{Si}=\mathrm{C}$ and $\mathrm{C}=\mathrm{C}$ bonds to provide a continuous honeycomb core structure for the molecular. The $\mathrm{C}=\mathrm{C}$ bond shortening is quite severe, which shows that the accommodation of the two types of bonds not to be a facile matter. The instability of $g$ - $\mathrm{SiC}_{7}$ can be understood in terms not only of the bond length changing but as a result of the alternating $\mathrm{Si}=\mathrm{C}$ and $\mathrm{C}=\mathrm{C}$ bonds governing the accommodation which causes a departure from the stable flatness, buckling the $g \mathrm{SiC}_{3}$ core. A summary of the total numbers of 
effectively unpaired electrons is indicated at each panel. As is seen in the figure, the value is quite large in all the cases supporting open-shell nature of the studied molecules. The quantity is peculiarly distributed over the molecule atoms as seen in left panels of Fig. 6. Evidently, the edge atoms with two dangling bonds each dominate in the distribution. Both silicon and carbon atoms are among the dominants.

To reveal what is going in the inner part of the molecules the edge atoms are terminated by hydrogens, which considerably inhibits the radicalization. Fig. 7 repeats the picture presented in Fig. 6 while influenced by the termination. As is seen in Fig. 7, on first glance, the termination does not affect the core structure much while $N_{D A}$ maps in the left panels disclose the influence by a considerable changing of their shape caused by the redistribution of $\mathrm{C}=\mathrm{C}$ and $\mathrm{Si}=\mathrm{C}$ lengths in due course of the termination. The $\mathrm{Si}=\mathrm{C}$ and $\mathrm{C}=\mathrm{C}$ bonds intervals become remarkably narrower, namely: $\{1.74-1.70\} \AA$ and $\{1.42-1.37\} \AA$ in $g \mathrm{SiC}_{3}$ while $\{1.69-1.67\} \AA$ and $\{1.47-1.38\} \AA$ in $g \mathrm{SiC}_{7}$. Nevertheless, the terminated $g \mathrm{SiC}_{7}$ remains buckled thus pointing to the consequence of $\mathrm{Si}=\mathrm{C}$ and $\mathrm{C}=\mathrm{C}$ bonds as main factor for comfortable flat accommodation. The difference in atom sizes and covalent bonds lays the foundation of the atom accommodation within a sheet, the flatness of which should be examined before DFT PBC modeling of new materials and not taken for granted as was done in [34-36]. Thus, $g$-SiC $\mathrm{S}_{7}$ is the same flake 'new material' as $s$ - $\mathrm{BNSi}_{2}$ discussed in the previous Section.

\section{UHF-Based Determination of $a^{\text {SO }}$ SOC Constant of Siliceous $s p^{2}$ Molecule}

In contrast with carbon counterparts, all silicon molecules, involving so called "Si=Si double bonds", do not exist and can be considered only virtually. Consequently, the evaluation of SOC parameters acquires a virtual character as well. To be in line with the procedure applied to carbonaceous species [25] we have selected fullerene $\mathrm{Si}_{60}$ and $(5,5)$ NSil molecule to look at the splitting of spin orbital energies when they are considered in the RHF and UHF approaches. Fig. 8 shows the result of the RHF and UHF application to a set of 28 spin orbitals in the vicinity of the HOMO-LUMO gaps of both molecules. When comparing it with the data for $\mathrm{C}_{60}$ and (5, 5) NGr [25], the outward effect in the cases of both carbon and silicon molecules is quite common. Thus, high degeneracy for the RHF states of both fullerenes, caused by their $I_{h}$ symmetry, is evidently removed. In the case of $(5,5)$ molecules it is less evident due to not so vivid degeneracy of the RHF states. As for the $a^{50}$ constant values, in the case of silicon molecules, they are obtained by following the Lande interval rule [25] and fill the interval of $9 \mathrm{meV}-194 \mathrm{meV}$ and $4 \mathrm{meV}-210 \mathrm{meV}$ for the considered spin orbitals of $\mathrm{Si}_{60}$ and $(5,5) \mathrm{NSil}$, respectively. As well as in the case of carbonaceous molecules, the values involve the energy splitting caused by lowered symmetry in the UHF states. To determine the magnitude of this contribution is not yet possible. If one takes into account the evaluation of splitting the energy band at $\mathrm{K}$ points of silicene crystal $(0.07 \mathrm{meV}-$ $1.55 \mathrm{meV}$ ) [37], the structure-induced contribution is quite considerable.

\section{A Few Comments about Germanene and Stanene}

Along with silicene, considerable attention has been paid lately to honeycomb structures, consisting of the next members of the tetrel family, namely, germanium and stannum [6,37-43]. For a long time the studies have been limited to DFT computations, supplemented by PBCs, of pencil-drawn structures presenting virtual germanene and stanene. As was found similarly to silicene, the electronic band structure of two latter crystals can be described in a quasirelativistic approach with the main attention to Dirac fermions. A joint picture of the band structure of graphene, silicene, germanene and stanene is shown in Fig. 9. The model structures are presented in the figure by similar honeycomb configurations. However, to fit the energy minimum, the flat structure of graphene should be buckled, even more so in the cases of germanene and stanene.

Empirical implementation of the associated 2D structures was achieved as epitaxial growth of corresponding monolayers on crystallographically suitable surface, namely on $\operatorname{Ag}(111), \operatorname{Pt}(111), \operatorname{Au}(111)$, and finally on $\mathrm{Al}(111)$ [41] and $\mathrm{Si}(111)$ [42] in the case of germanene and on $\mathrm{Bi}_{2} \mathrm{Te}_{3}(111)$ in the case of stanene [43]. In all the cases the choice of the relevant substrate has been done on the basis of knowledge accumulated in the field of surface science. All the chosen substrates were able to promote hexagonal atom packing in the corresponding adlayers that provided Dirac cone structure of the related electronic bands.

The successful realization of epitaxial germanene and stanene does not match the validity of structure and electronic properties of the relevant virtual crystals. If in the case of silicene one could speak about a likeness of atomic structure of virtual silicene molecule and epitaxial silicene, although even in this case the structure of the molecule (see insert in Fig. 9b) still significantly differs from both flat and curved crystal structures (Fig. 1a). It is not actually the case of germanene and stanene. The matter is not about a pencil-drawn virtual species, but about stability of a honeycomb configuration for germanene and stanene molecules that was already discussed for 

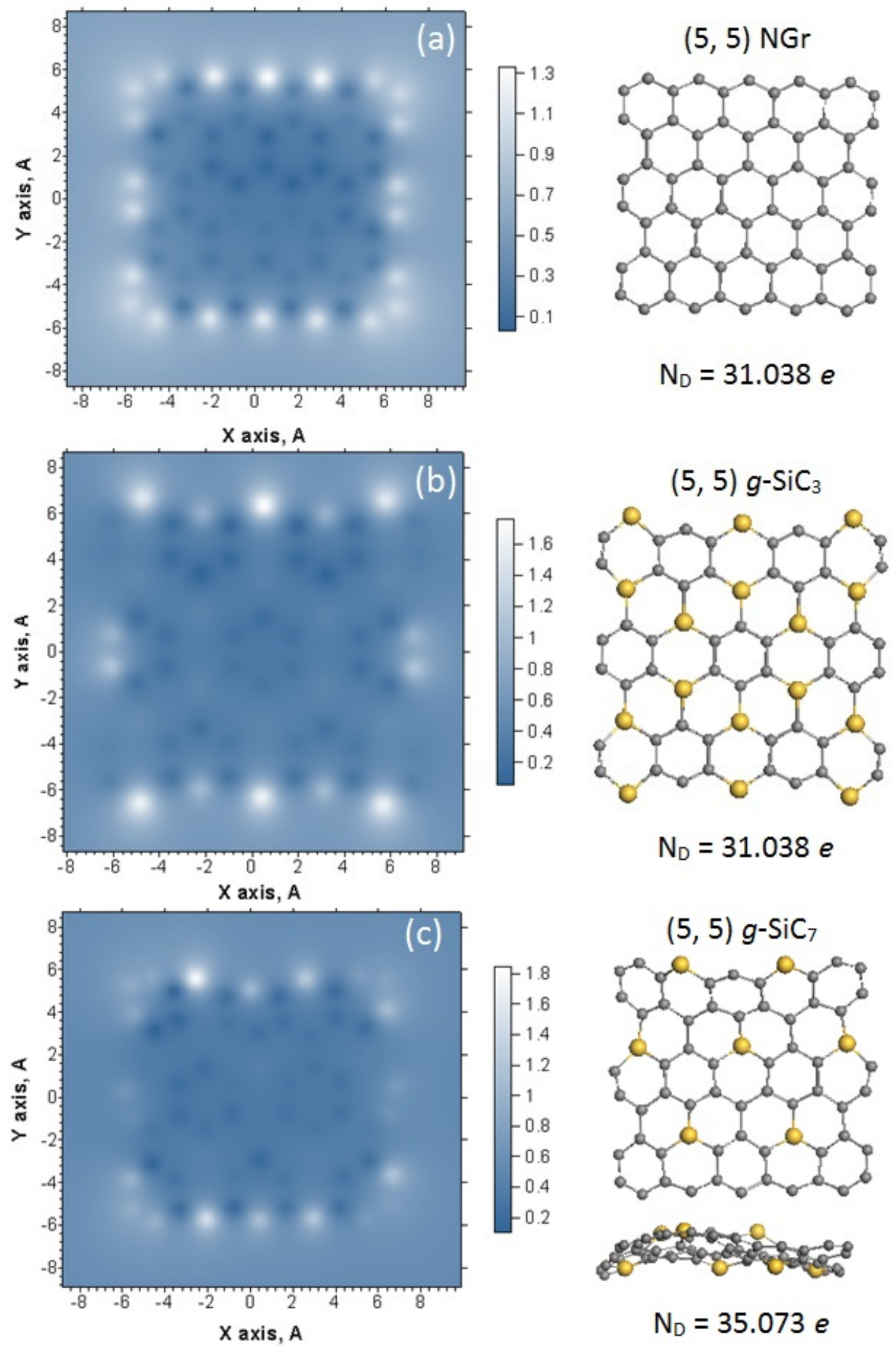

FIG. 6. Equilibrium structures (right panels) and $N_{D A}$ maps (left panels) of bare $(5,5) \mathrm{Ngr}$ (a), $(5,5){\mathrm{g}-\mathrm{SiC}_{3}}_{3}$ (b) and $(5,5) \mathrm{g}-\mathrm{SiC}_{7}$ (top and side views) (c) molecules. Scales show the amplitude of the $N_{D A}$ values changing. UHF AM1 calculations. Small gray and big-light gray balls mark carbon and silicon, atoms, respectively 

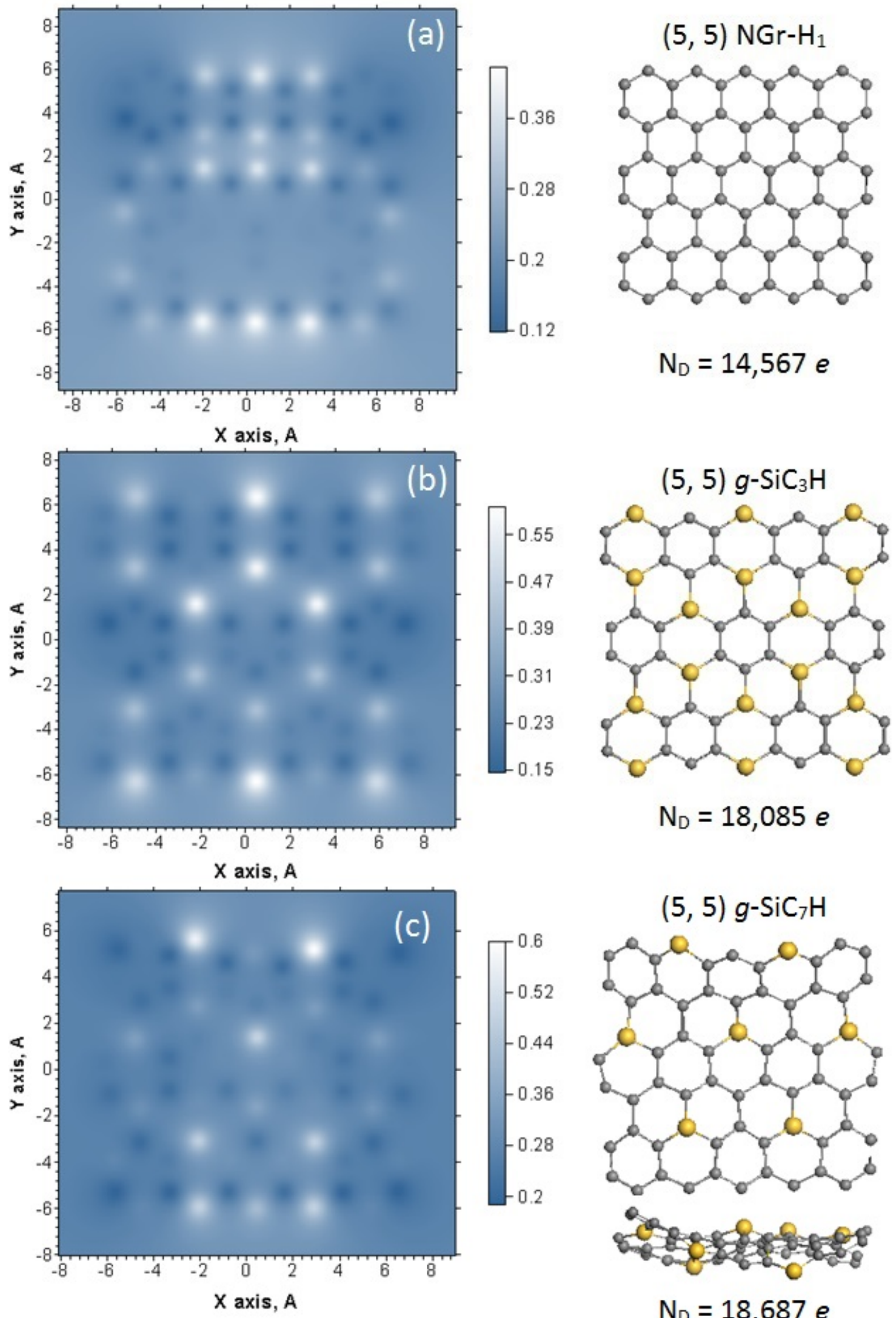

$(5,5) g-\mathrm{SiC}_{7} \mathrm{H}$

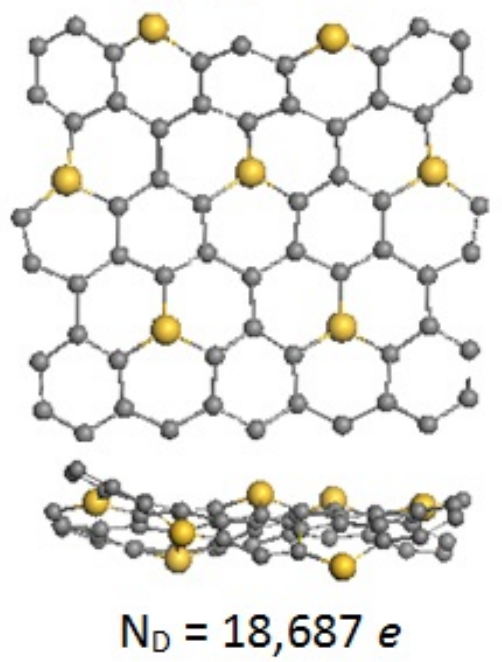

FIG. 7. Equilibrium structures (right panels) and $N_{D A}$ maps (left panels) of hydrogen-terminated $(5,5) \mathrm{Ngr}(\mathrm{a}),(5,5) g-\mathrm{SiC}_{3}$ (b) and $(5,5) g-\mathrm{SiC}_{7}$ (top and side views) (c) molecules. Scales show the amplitude of the $N_{D A}$ values changing. Hydrogen atoms are not shown. UHF AM1 calculations. Atom marking see in the caption to Fig. 6 


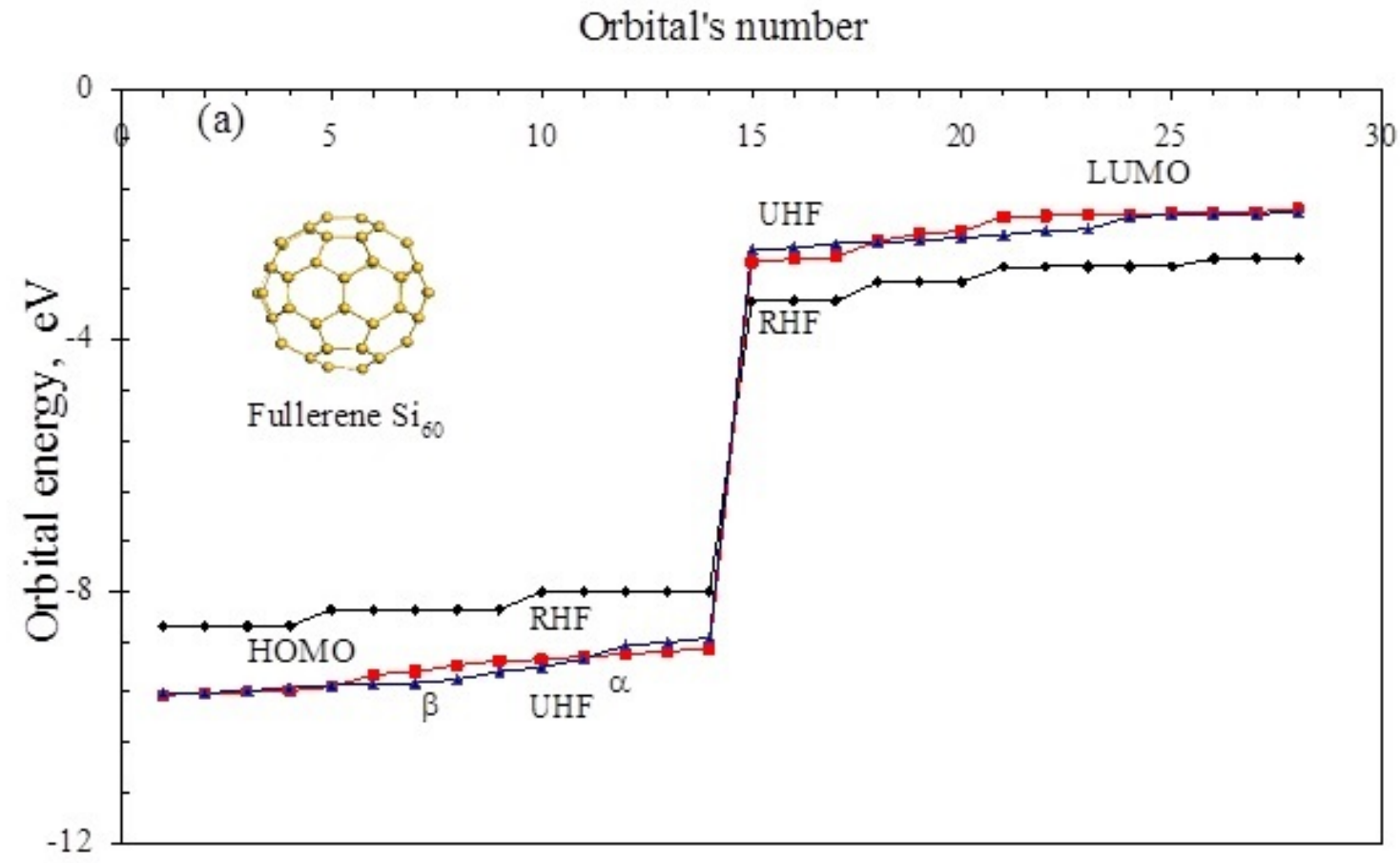

Orbital's number

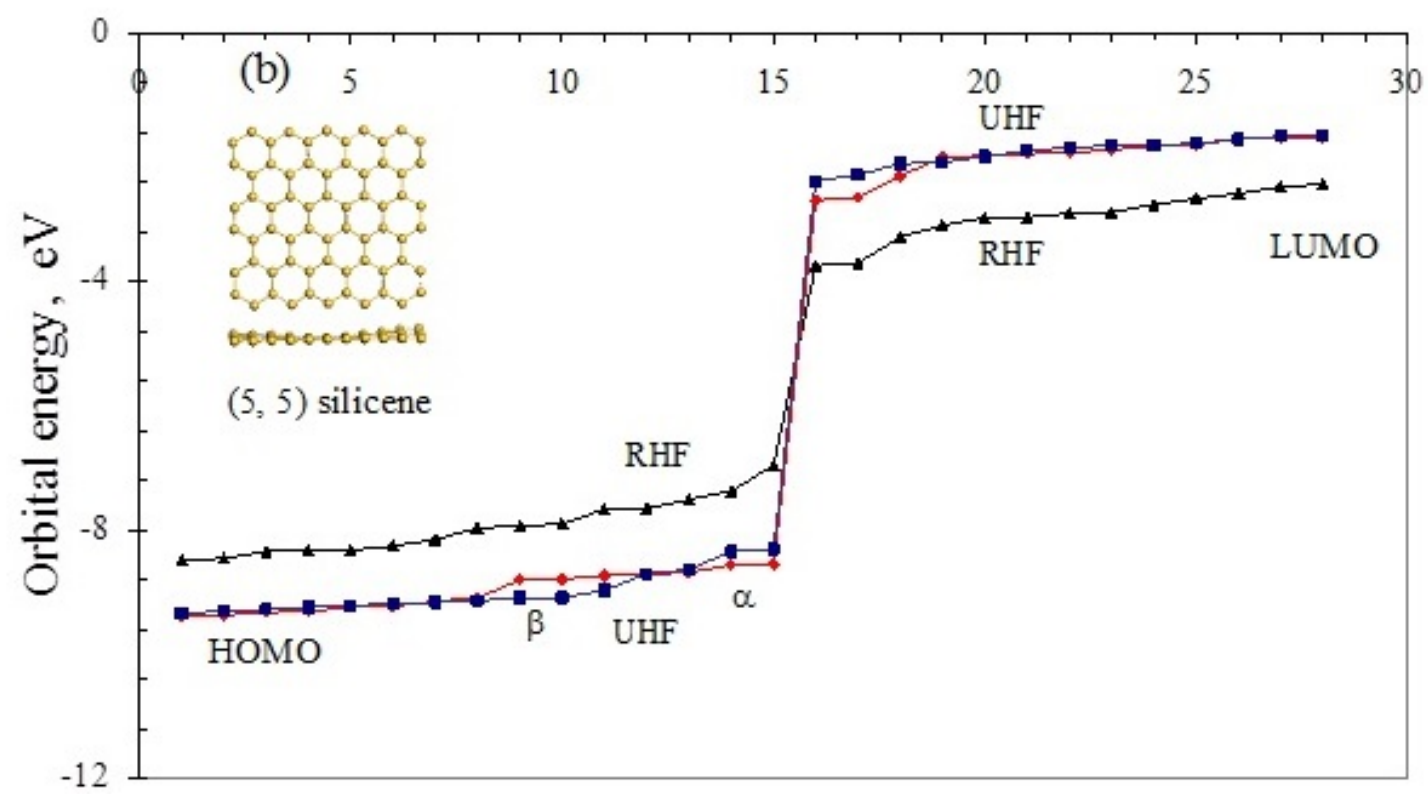

FIG. 8. Energies of 25 spin orbitals in the vicinity of HOMO-LUMO gaps of fullerene $\mathrm{Si}_{60}$ (a) and $(5,5)$ NSil molecule (b). UHF AM1 calculations 

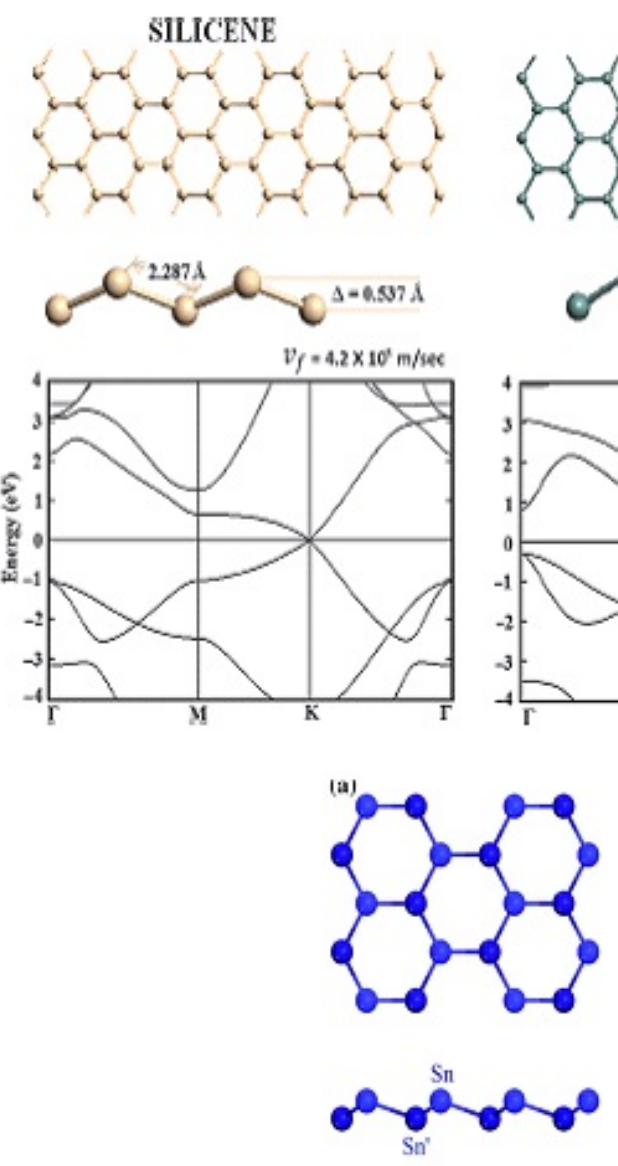

GERMANENE
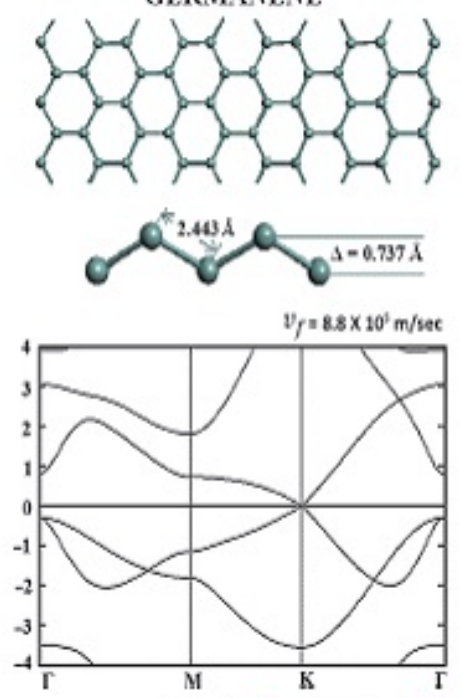

GRAPHENE
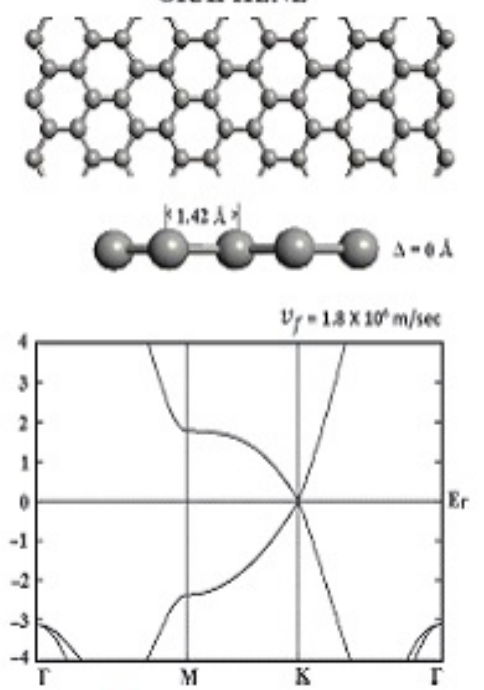

(a)

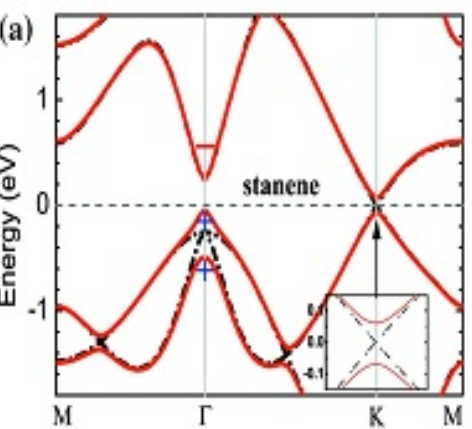

FIG. 9. Structure, band-structure with Fermi velocities of silicene, germanene and graphene (adapted from [39] and [41])

$s$-BNSi ${ }_{2}$. To clarify this point, let us look at single-hexagon and multi-hexagon structures of the tetrel family atoms.

Figure 10 presents UHF equilibrium structures of benzene-like $X_{6} H_{6}(\mathrm{X}=\mathrm{C}, \mathrm{Si}, \mathrm{Ge}$, and $\mathrm{Sn}$ ) molecules while Table 2 accumulates their structural and radicalization parameters. The benzene-like pattern is characteristic for all the molecules, absolutely flat in case of $\mathrm{C}_{6} \mathrm{H}_{6}$ and $\mathrm{Si}_{6} \mathrm{H}_{6}$ while somewhat out of planarity for $\mathrm{Ge}_{6} \mathrm{H}_{6}$ and $\mathrm{Sn}_{6} \mathrm{H}_{6}$. In the latter case, the benzene-like configuration is energetically less favorable (by $25 \%$ ) comparing with the boat-like configuration shown in Figs. 10e-f. All the benzene-like molecules are characterized by uniform bond length while there are two bond lengths in the boat-like $\mathrm{Sn}_{6} \mathrm{H}_{6}$ molecule. The transition from closed-shell to open-shell behavior is marked by the $R_{c o v}^{d b}$ [25] values which are given in Table 2 as well. When $R_{e q}^{d b}<R_{c o v}^{d b}$, the relevant molecule in the ground state is a closed-shell one. According to the table, this concerns $G_{6} e_{6}$ and one of $\mathrm{Sn}_{6} \mathrm{H}_{6}$ molecules, which explains why the molecules are not radicalized $\left(N_{D}=0\right)$ similarly to $C_{6} H_{6}$ for which $R_{e q}^{d b} \cong R_{c o v}^{d b}$. Therefore, only in $S i_{6} H_{6}$ molecule all the bonds are radicalized due to $R_{e q}^{d b}>R_{c o v}^{d b}$ as well as two longer bonds of the boat-like $\mathrm{Sn}_{6} \mathrm{H}_{6}$ molecule for the same reason.

Following this brief analysis of the structural and radical character of the $X_{6} H_{6}$ molecules, one finds both similarity and difference of the species at the basic level. Obviously, similarity inspires hope to obtain silicene, germanene and stannene as prospective new-material playground of the around-graphene science. The similarity excuses a voluntary choice of the majority of computationists to take the flat honeycomb structure of graphene as the basic tetrene DFT PBC models. At the same time, the difference between the molecules casts doubt on the soundness of the basic model choice. Let us see how these concerns are valid.

Figure 11 presents the results for the optimization of the preliminary equi-structural honeycomb compositions of $X_{66}$ tetrenes. The configuration corresponds to a rectangular $(5 \times 5)$ nanoX fragment that involves five hexagons along armchair and zigzag directions, respectively. The equilibrium structures are presented in top and side projections. As seen in the figure, carbon and silicon compositions preserve the honeycomb structure, perfectly 
TABLE 2. $\mathrm{X}=\mathrm{X}$ bond lenght, $\AA$, and molecular chemical susceptibility $\left(N_{D}\right), e$ in the benzenelike and $(5 \times 5)$ honeycomb $\left(X_{66}\right)$ tetrenes

\begin{tabular}{|c|c|c|c|c|c|}
\hline \multicolumn{2}{|c|}{ Molecule } & $\mathrm{C}$ & $\mathrm{Si}$ & $\mathrm{Ge}$ & $\mathrm{Sn}$ \\
\hline \multirow{3}{*}{$\mathrm{X}_{6} \mathrm{H}_{6}$} & $R_{e q}^{d b}$ & 1.395 & 2.293 & 2.026 & $2.544(4), 2.593(2)^{*}, 2.256^{* *}$ \\
\cline { 2 - 6 } & $R_{c o v}^{d b}$ & 1.395 & 1.80 & 2.10 & 2.55 \\
\cline { 2 - 6 } & $N_{D}$ & 0.05 & 2.68 & 0 & $1.030^{* *}$ \\
\hline $\mathrm{X}_{66-}$ & $R_{e q}^{d b}$ & $1.291-1.469^{* * *}$ & $2.214-2.330^{* * *}$ & $1.941-2.407^{* * *}$ & $2.023-2.709^{* *}$ \\
\cline { 2 - 6 }$(5 \times 5)$ nanoX & $N_{D}$ & 16.63 & 42.51 & 5.56 & 10.96 \\
\hline
\end{tabular}

${ }^{*}$ The shortest and longest bonds of the molecules in Fig. 10e and Fig. 10d.

${ }^{* *}$ The data related to the molecule in Fig. $10 \mathrm{~g}$.

*** The data are related to equilibrium structures in Fig. 11
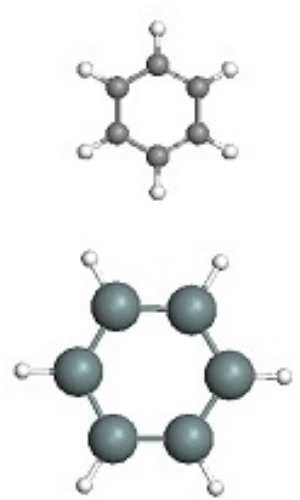

(a)

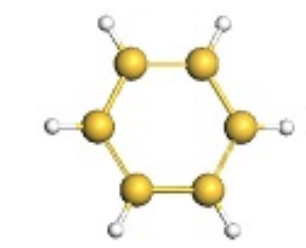

(d)

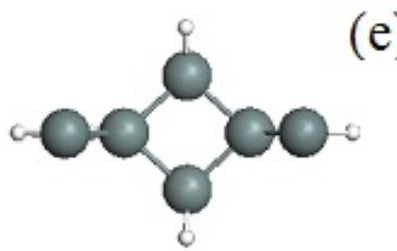

(b)

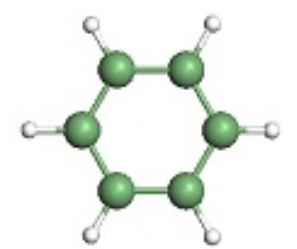

(c)

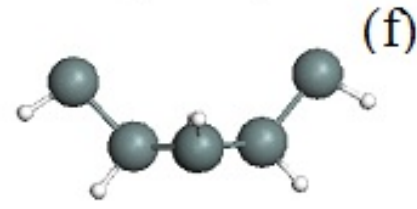

FIG. 10. Equilibrium structure of $X_{6} H_{6}$ molecules when $\mathrm{X}=\mathrm{C}(\mathrm{a}), \mathrm{Si}(\mathrm{b})$, Ge (c), and $\mathrm{Sn}$ (d-f). In the latter case, benzene-like (d) and boat-like (top (e) and side (f) views) compositions are presented. Gray, light-gray, big gray, steel-gray, and white balls mark carbon, silicon, germanium, tin, and hydrogen atoms. The balls' sizes roughly correspond to the relevant van der Waals diameters. UHF AM1 for $\mathrm{X}=\mathrm{Si}$, Ge and UHF PM3 for $\mathrm{X}=\mathrm{Sn}$ calculations (adapted from [25])

planar in the former case and of slightly violated planarity in the latter. In both cases, the $\mathrm{X}=\mathrm{X}$ bond length values are quite dispersed and occupy interval, the limit values of which are given in Table 2 . The presence of bonds, the lengths of which exceed $R_{c o v}^{d b}$, provides a considerable radicalization of the fragments $\left(N_{D}\right)$ in both cases.

A detailed radicalization of the two honeycomb structures $C_{66}([5,5] \mathrm{NGr})$ and $S i_{66}([5,5] \mathrm{NSil})$ are presented by $N_{D A}$ maps in Fig. 12. As is seen in the figure, 22 edge atoms dominate in both distributions. Their positions do not coincide due to the different numeration of atoms in the model molecules. The radicalization of graphene does not prevent it from existing under ambient conditions because the latter is mainly concentrated on the circumference and thus is usually well inhibited by the termination of edge atoms. As for silicene, the termination of edge atoms is not enough to inhibit its high radicalization since the latter remains still high on the atoms in basal plane as well due to which free standing one-atom thick silicene sheet cannot exist under ambient conditions. Actually, as noted in numerous publications, epitaxial silicene is highly chemically active [7] and can survive in UHV only. Once designed for a practical application under ambient conditions, say, for field-effect transistors it should be laminated by thin $\mathrm{Al}$ or $\mathrm{AlO}_{2}$ films [18]. It is worthwhile to remember a deep comment of Roald Hoffmann concerning the chemical peculiarity of silicene [38]: “... There is a growing literature out there of the Si analogue, silicene... And that literature talks about silicene as if it were graphene. In part this is an attempt to live off graphene's mystique, but part comes out of lack of knowledge of chemistry... I don't often say something categorical, but I will say that a pristine free standing single layer sheet of silicene (or a Si nanotube) will not be made. Silicene exists and will be made only on a support of some sort, metal or semiconductor. The reason for this is, of course, the well-known kinetic and energetic instability of $\mathrm{Si}-\mathrm{Si}$ double bonds... They will latch on chemically to every piece of molecular dirt around." 
(a)

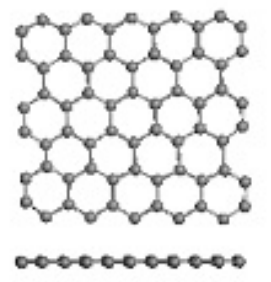

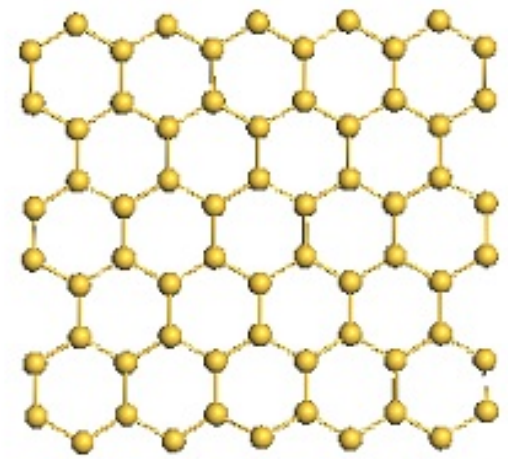

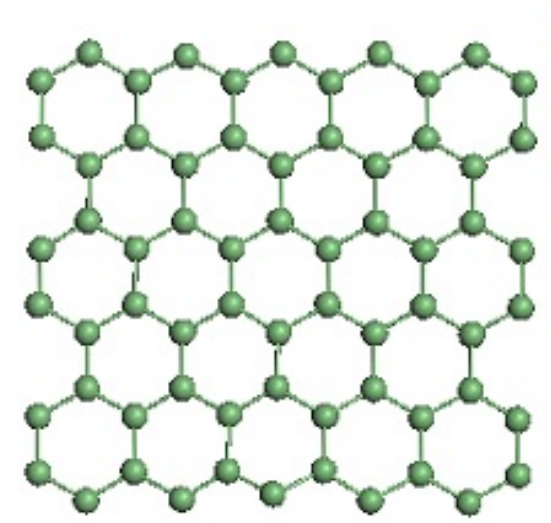

(c)
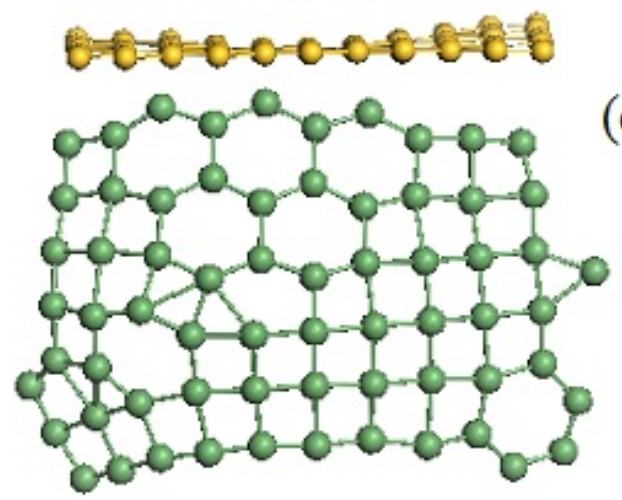

(d)

(e)
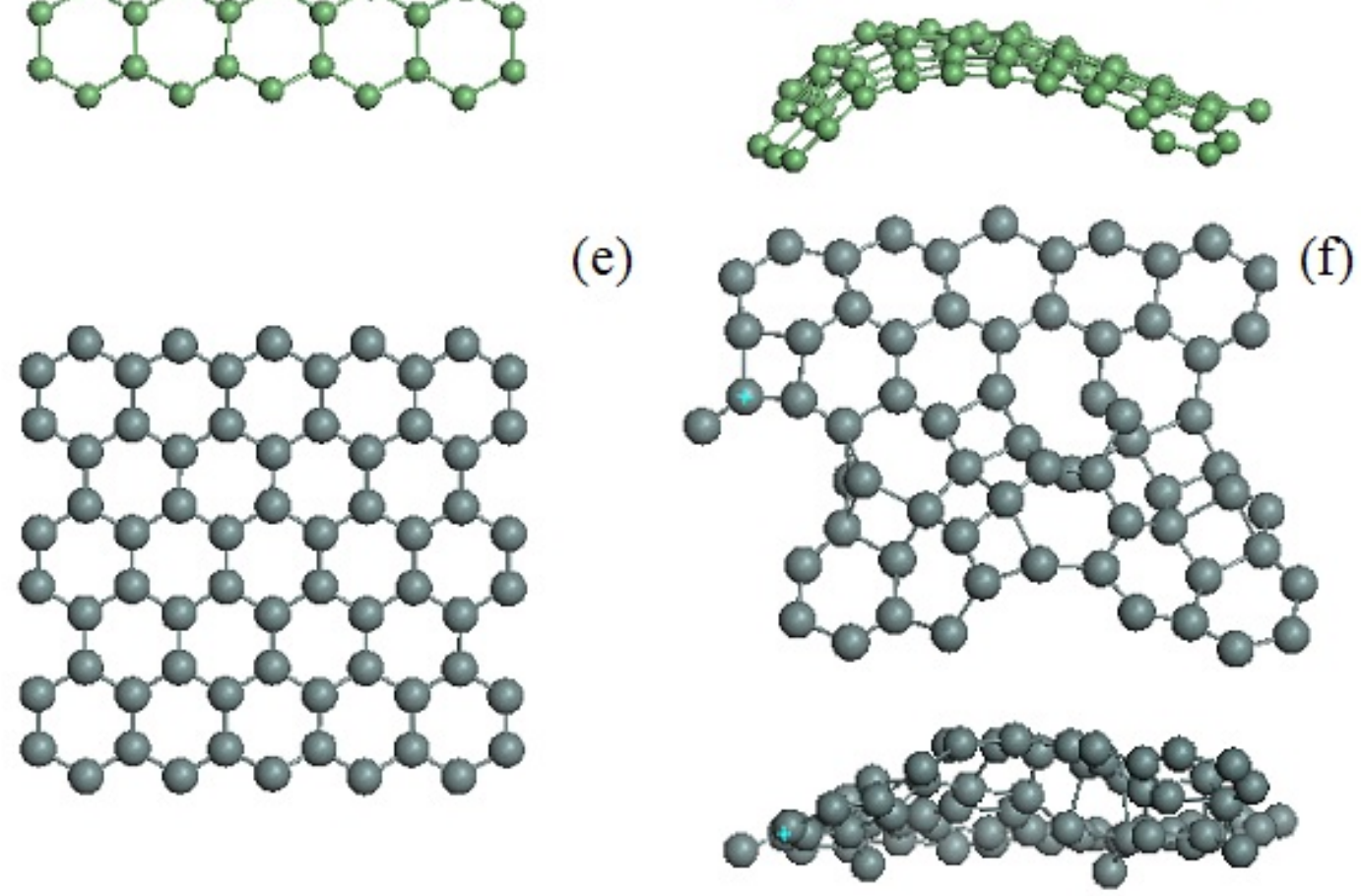

FIG. 11. Equilibrium structure of $X_{66}(5 \times 5)$ nanoX honeycomb fragments. (a) $\mathrm{C}_{66}$; (b) top and side views of $\mathrm{Si}_{66}$; (d) top and side views of $\mathrm{Ge}_{66}$; (f) top and side views of $\mathrm{Sn}_{66}$. (c) and (e) $\mathrm{Ge}_{66}$ and $\mathrm{Sn}_{66}$ start configurations, respectively. Atom marking see in the caption to Fig. 10. The balls' sizes roughly correspond to the relevant van der Waals diameters. UHF AM1 and UHF PM3 calculations for $\mathrm{X}=\mathrm{C}, \mathrm{Si}, \mathrm{Ge}$ and $\mathrm{X}=\mathrm{Sn}$, respectively (adapted from [25]) 


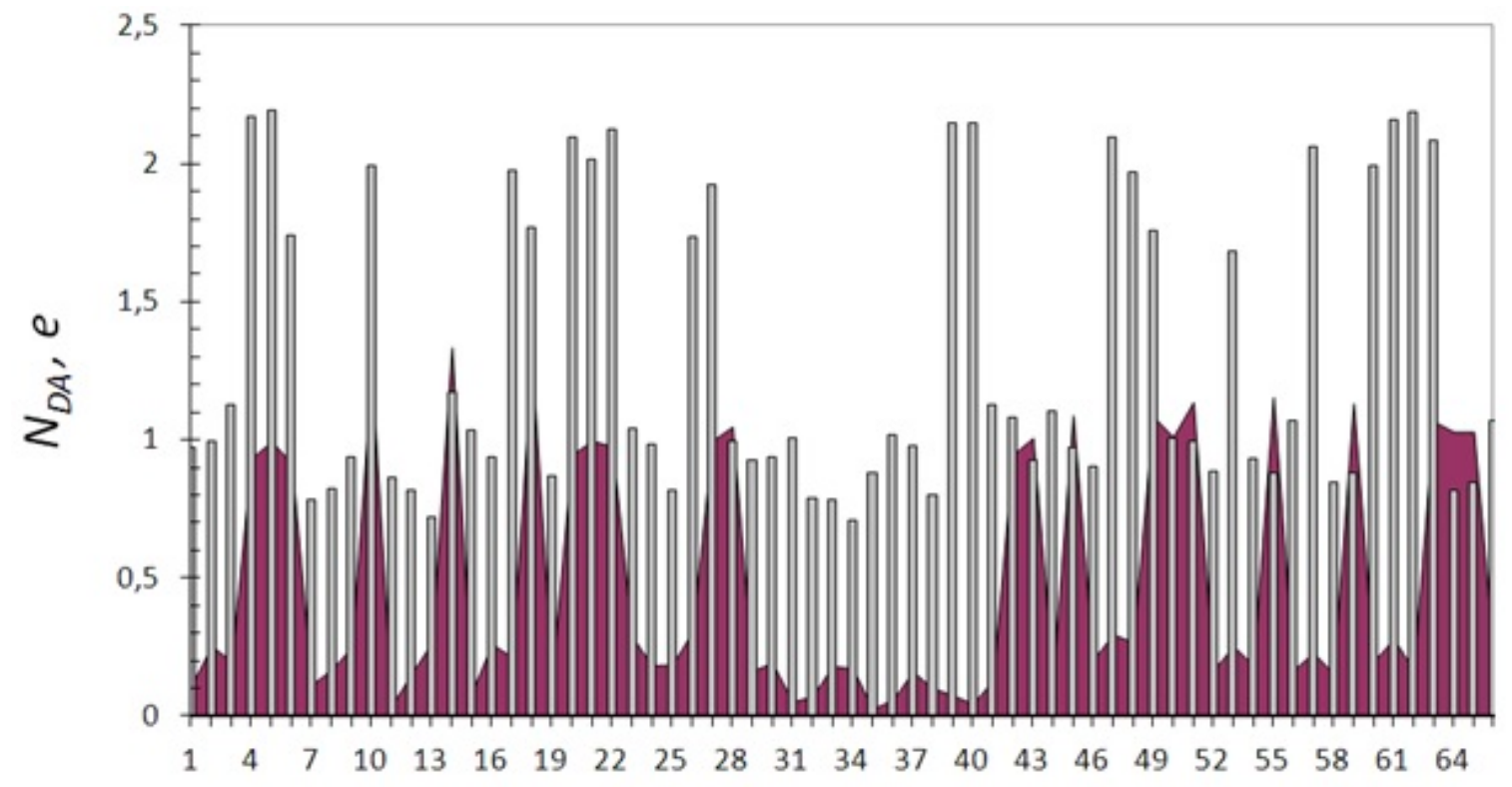

Atom number

FIG. 12. $N_{D A}$ distribution over atoms of bare $(5,5)$ NGr (filled area) and $(5,5)$ NSil (bars) molecules. UHF AM1 calculations

In contrast to the above species, $X_{66}$ tetrenes of germanium and tin do not preserve the honeycomb structure in due course of the optimization. Their initial structures in Fig. 11c and Fig. 11e are just replicas of the equilibrium structure of $S i_{66}$ in Fig. 11b. The followed optimization drastically disturbs the structures leaving only small clusters of condensed hexagon rings and making them considerably non planar thus showing an obvious quantum instability of the $2 \mathrm{D}$ species similar to that discussed for $(5,5) s-\mathrm{BNSi}_{2}$. The bond lengths cover much wider interval, abandoned with short bonds, for which $R_{e q}^{d b}<R_{c o v}^{d b}$. The latter explains why the total radicalization of both fragments is less than in the case of $C_{66}$ and $S i_{66}$ (see Table 2). Possibly, such a large dispersion of the $\mathrm{X}=\mathrm{X}$ bond length resulted from the extended isomerism of the species, which is characteristic for Ge- and Sn-tetrenes in contrast to $\mathrm{C}$ - and Si-ones, lays the foundation of the quantum instability.

According to the data presented in Fig. 11 and Table 2, the high radicalization and quantum instability of the honeycomb $2 \mathrm{D}$ structure are two main reasons that greatly complicate the existence of higher tetrene materials in practice. The former is mainly related to $S i_{66}$ fragments while the latter concerns $G e_{66}$ and $S n_{66}$. As for germanene and stanene, the data from Table 2 tell that the fragment radicalization is much lower than for graphene and cannot be considered as the main difficulty for the species existence. However, until now none of numerous attempts to obtain free standing either germanene or stanene in practice has been successful. The inability of the tetrene atoms to form a lengthy honeycomb structure is apparently the major deterrent due to which the formation of the desired free standing sheets is not achievable. As we see above, this obstacle was overcome by the choice of a suitable substrate surface on which the adsorbed tetrels can form hexagon-patterned structures.

\section{Conclusion}

Carbon is the first member of the tetrel family of group 14 atoms of Mendeleev's table and the outstanding importance of $C \leftrightarrow C$ bonds for organic chemistry is very stimulating for looking for a similar behavior of $X \leftrightarrow X$ chemical bonds formed by heavier tetrels. The similarity and dissimilarity of different members of the family have been the topic of heated discussions for more than a century. The current science regarding graphene represents a new milestone of activity in this direction and is full of suggestions for new prototypes of graphene the foremost of which are based on the equivalent-electron atoms such as silicon, germanium and stannum. However, for formally implemented in PBC honeycomb structures, these species suffer from high radicalization and quantum instability leaving graphene and partially graphene-based materials the only representative of one-atom-thick 2D crystals. 


\section{Acknowledgements}

The author is grateful to P.V. Avramov for fruitful discussions. The work was performed under financial support of the Peoples' Friendship University of Russia, grant: 022203-0-000.

\section{References}

[1] Takeda K., Shiraishi K. Theoretical possibility of stage corrugation in Si and Ge analogs of graphite. Phys. Rev. B, 1994, 50, P. 1491614922.

[2] Guzman-Verri G., Lew Yan Voon L.C. Electronic structure of silicon-based nanostructures. Phys. Rev. B, 2007 , 76, P. 075131.

[3] Kara A., Enriquez H., Seitsonen A.P., Voon L.L.Y., Vizzini S., Aufray B., Oughaddou H. A review on silicene - new candidate for electronics. Surf. Sci. Rep., 2012, 67, P. 1-18.

[4] Yamada-Takamura Y., Friedlein R. Progress in the materials science of silicene. Sci. Technol. Adv. Mater., 2014, 15, P. 064404(12pp).

[5] Voon L.L.I., Guzmàn-Verri G.G. Is silicene the next graphene? MRS Bull, 2014, 39, P. 366-373.

[6] Bhimanapati G.R., Lin Z., Meunier V., Jung Y., Cha J., Das S., Xiao D., Son Y., Strano M.S., Cooper V.R., Liang L., Louie S.G., Ringe E., Zhou W., Sumpter B.G., Terrones H., Xia F., Wang Y., Zhu J., Akinwande D., Alem N., Schuller J.A., Schaak R.E., Terrones M., Robinson J.A. Recent advances in two-dimensional materials beyond graphene. ACS Nano, 2015, 22, P. $12168-12173$.

[7] Oughaddou H., Enriquez H., Tchalala M., Yildirim H., Mayne A., Bendounan A., Dujardin G., Ali M., Kara A. Silicene, a promising new 2D material. Prog. Surf. Sci., 2015, 90, P. 46-83.

[8] Lew Yan Voon L.C. Zhu J. Schwingenschloechloel U. Silicene: Recent teoretical advances. Appl. Phys. Rev.,2016, 3, P. 040802.

[9] Kara A., Léandri C., Dávila M.E., De Padova P., Ealet B., Oughaddou H., Aufray B., Le Lay G. Physics of silicene stripes. J. Supercond Nov. Magn., 2008/2009, 22, P. 259-263.

[10] Sheka E.F. May silicene exist?, 2009. arXiv:0901.3663 [cond-mat.mtrl-sci].

[11] Vogt P., De Padova P., Quaresima C., Avila J., Frantzeskakis J., Asensio M.C., Resta A., Ealet B., Le Lay G. Silicene: Compelling experimental evidence for graphenelike two-dimensional silicon. Phys. Rev. Lett., 2012, 108, P. 155501.

[12] Lin C.-N., Arafune R., Kawahara R., Tsukahara N., Minamitani E., Kim Y., Takagi N., Kawai N. Structure of silicene grown on Ag(111). Appl. Phys. Express, 2012, 5, P. 045802.

[13] Chiappe D., Grazianetti C., Tallarida G., Fanciulli M., Molle A. Local electronic properties of corrugated silicene phases. Adv. Mater., 2012, 24, P. 5088-5093.

[14] Chen L., Liu C.C., Feng B., He X., Cheng P., Ding Z., Meng S., Yao Y.G., Wu K.H. Evidence for Dirac fermions in a honeycomb lattice based on silicon. Phys. Rev. Lett., 2012, 109, P. 056804.

[15] Fleurence A., Friedlein R., Ozaki T., Kawai H., Wang Y., Yamada-Takamura Y. Experimental evidence for epitaxial silicene on diboride thin films. Phys. Rev. Lett., 2012, 108, P. 245501.

[16] Meng L., Wang Y., Zhang L., Du S., Wu R., Li L., Zhang Y., Li G., Zhou H., Hofer W.A., Gao H.J. Buckled silicene formation on Ir(111). Nano Lett., 2013, 13, P. 685-690.

[17] Zhong H.-X., Quhe R.-G., Wang Y.-Y., Shi J.-J., Lü J. Silicene on substrates: A theoretical perspective. Chin. Phys. B, 2015, 24, P. 087308.

[18] Tao L., Cinquanta E., Chiappe D., Grazianetti C., Fanciulli M., Dubey M., Molle A., Akinwande D. Silicene field-effect transistors operating at room temperature. Nat. Techn., 2015, 10, P. 227-231.

[19] Sheka E.F. Why $\mathrm{sp}^{2}$-like nanosilicons should not form: Insight from quantum chemistry. Int J. Quant. Chem., 2013, 113, P. 612-618.

[20] Liu C.-C., Feng W., Yao Y. Quantum spin Hall effect in silicene and two-dimensional germanium. Phys. Rev. Lett., 2011 , 107, P. 076802.

[21] Cahangirov S., Topsakal M., Aktürk E., Şahin H., Ciraci S. Two- and one-dimensional honeycomb structures of silicon and germanium. Phys. Rev. Lett., 2009, 102, P. 236804.

[22] Avramov P., Demin V., Luo M., Choi C.H., Sorokin P.B., Yakobson B., Chernozatonskii L. Translation symmetry breakdown in lowdimensional lattices of pentagonal rings. J. Phys. Chem. Lett., 2015, 6, P. 4525-4531.

[23] Sheka E.F. Fullerenes: Nanochemistry, Nanomagnetism, Nanomedicine, Nanophotonics. CRC Press, Taylor and Francis Group: Boca Raton, 2011.

[24] Sheka E.F. Spin-orbit concept of open-shell systems, 2015. arXiv: 1511:05483 [physics.chem-ph].

[25] Sheka E.F. Stretching and breaking of chemical bonds, correlation of electrons, and radical properties of covalent species. Adv. Quant. Chem., 2015, 70, P. 111-161.

[26] Morkin T.L., Owens T.R., Leigh W.J. Kinetic studies of the reactions of $\mathrm{Si}=\mathrm{C}$ and Si-Si bonds. in The Chemistry of Organic Silicon Compounds, ed. Z. Rappoport and Y. Apeloig, John Wiley \& Sons, Chichester, 2001, Vol. 3, P. 949-1026.

[27] Karni M., Kapp J., Schleyer P.R., Apeloig Y. Theoretical aspects of compounds containing Si, Ge, Sn and Pb. in The Chemistry of Organic Silicon Compounds, ed. Z. Rappoport and Y. Apeloig, John Wiley \& Sons, Chichester, 2001, Vol. 3, P. 1-164.

[28] Wang S. A comparative first-principles study of orbital hybridization in two-dimensional C, Si, and Ge. Phys. Chem. Chem. Phys., 2011, 13, P. 11929-11938.

[29] Sheka E.F., Chernozatonskii L.A. Bond length effect on odd electrons behavior in single-walled carbon nanotubes. J. Phys. Chem. A, 2007, 111, P. 10771.

[30] Fucutome H. Unrestricted Hartree-Fock theory and its applications to molecules and chemical reactions. Int. J. Quant. Chem., 1981, 20, P. 955-1065.

[31] Andriotis A.N., Richter E., Menon M. Prediction of a new graphenelike Si2BN solid. Phys. Rev. B, 2016, 93, P. 081413(R).

[32] Aselage T.L. The coexistence of silicon borides with boron-saturated silicon: Metastability of SiB 3. J. Mat. Res., 1998, 13, P. 1786-1794.

[33] Hoffmann R. Small but strong lessons from chemistry for nanoscience. Angew Chem. Int. Ed., 2012, 51, P. 2-13.

[34] Zhao M., Zhang R. Two-dimensional topological insulators with binary honeycomb lattices: $\mathrm{SiC}_{3}$ siligraphene and its analogs. Phys. Rev. $B, 2014,89$, P. 195427.

[35] Shi Z., Zhang Z., Kutana A., Yakobson B.I. Predicting two dimensional silicon carbide monolayers. ACS Nano, 2015 , 9, P. $9802-9809$.

[36] Dong H., Zhou L., Frauenheim T., Hou T., Lee S.-T., Li Y. SiC 7 siligraphene: Novel donor material with extraordinary sunlight absorption. Nanoscale, 2016, 8, P. 6994-6999. 
[37] Balendhran S., Walia S., Nili H., Sriram S., Bhaskaran M. Elemental analogues of graphene: silicene, germanene, stanene, and phosphorene. Small, 2015, 11, P. 640-652.

[38] Trivedi S., Srivastava A., Kurchania R. Silicene and germanene: A first principle study of electronic structure and effect of hydrogenationpassivation. J. Comp. Theor. Nanosci., 2014, 11, P. 781-788.

[39] Xu Y., Yan B., Zhang H.-J., Wang J., Xu G., Tang P., Duan W., Zhang S.-C. Large-gap quantum spin Hall insulators in tin films. Phys. Rev. Lett., 2013, 111, P. 136804

[40] Cai B., Zhang S., Hu Z., Hu Y., Zoua Y. Zeng H. Tinene: a two-dimensional Dirac material with a 72 meV band gap. Phys. Chem. Chem. Phys., 2015, 17, P. 12634-12638.

[41] Derivaz M., Dentel D., Stephan R., Hanf M.-C., Mehdaoui A., Sonnet P., Pirri C. Continuous germanene layer on Al(111). Nano Lett., 2015, 15, P. 2510-2516.

[42] Tsai H.-S., Chen Y.-Z., Medina H., Su T.-Y., Chou T.-S., Chen Y.-H., Chueh Y.-L., Liang J.-O. Direct formation of large-scale multi-layered germanene on Si substrate. Phys. Chem. Chem. Phys., 2015, 17, P. $21389-21393$.

[43] Zhu F.-F., Chen W.-J., Xu Y., Gao C.-1., Guan D.-D., Liu C.-H., Qian D., Zhang S.-C., Jia J.-F. Epitaxial growth of two-dimensional stanene. Nat. Mat., 2015, 14, P. 1020-1025. 